\title{
Synthesis of Complex Thermally Coupled Distillation Systems Including Divided Wall Columns.
}

\begin{tabular}{|r|l|}
\hline Journal: & AIChE Journal \\
\hline Manuscript ID: & Draft \\
\hline Watey - Manuscript type: & Research Article \\
\hline Complete List of Authors: & $\begin{array}{l}\text { Caballero, Jose Antonio; Universidad de Alicante, Departamento de } \\
\text { Ingenieria Quimica } \\
\text { Grossmann, Ignacio; Carnegie Mellon University, Department of Chemical } \\
\text { Engineering }\end{array}$ \\
\hline Keywords: & Distillation, Mathematical modeling, Optimization, Process synthesis \\
\hline & \\
\hline
\end{tabular}

SCHOLARONE $^{\text {m }}$

Manuscripts 
Synthesis of Complex Thermally Coupled Distillation Systems Including Divided Wall Columns.

\author{
José A. Caballero*; Ignacio E. Grossmann ${ }^{* *}$ \\ *Department of Chemical Engineering. University of Alicante. Ap. Correos 99. 03080 Alicante. Spain \\ ** Department of Chemical Engineering. Carnegie Mellon University. Pittsburgh, PA. USA.
}

\begin{abstract}
.
This paper deals with the design of thermally coupled distillation sequences explicitly including the possibility of divided wall columns (DWC). A DWC with a single wall can be considered thermodynamically equivalent to a fully thermally coupled sub-system formed by three separation tasks (a Petlyuk configuration in the case of three component mixtures). In this paper it is shown how to systematically identify all the sequences of separation tasks that can produce configurations that include at least a DWC. Feasible sequences that explicitly include DWCs are enforced through a set of logical relationships in terms of boolean variables. These logical relationships include as feasible alternatives from conventional columns (each column must have a condenser and a reboiler) to fully thermally coupled systems (only one reboiler and one condenser in the entire system). A comprehensive disjunctive programming formulation for finding the optimal solution is presented. The model is based on the Fenske, Underwood Gilliland equations. However, the disjunctive formulation allows easily the use of any other shortcut, aggregated or even rigorous model without modifying much the structure of the model. Two illustrative examples illustrate the procedure. Finally, some general comments on the extension of the methodology to multi-wall columns are included.
\end{abstract}

\title{
Keywords
}

Distillation; Thermally coupled distillation; MINLP; Divided Wall Column; Superstructure optimization; GDP.

\section{Introduction}

Distillation is one of the most studied unit operations in chemical engineering. This effort is amply justified because distillation is the most widely used separation technique in process industries, and this trend is unlikely to change in the near future. In order to get an idea of the importance of distillation, Humphrey ${ }^{1}$ estimated that in the United States there are 40,000 distillation columns in operation that handle more than $90 \%$ of separations and purifications. The capital investment for these distillation systems is estimated to be 8 billion US\$. Using data by Mix et $\mathrm{al}^{2}$, Soave \& Feliu ${ }^{3}$ calculated that distillation accounts about the $3 \%$ of the total US energy consumption, which is equivalent to $2.87 \times 10^{18} \mathrm{~J}$ (2.87 million TJ) per year, or to a power consumption of $91 \mathrm{GW}$, or 54 million tons of crude oil.

The distillation research is motivated by its major limitations. Distillation columns use very large amounts of energy because the evaporation steps involved. Typically more than half of the 
process heat distributed to a plant is dedicated to supply heat in the reboilers of distillation columns ${ }^{4}$. Unfortunately, this enormous amount of energy is introduced in the bottom of the column and approximately the same amount of energy removed in the top, but at significantly lower temperature, which renders a very inefficient process -but also one of the most effective, for separation mixtures. Although distillation research is often regarded as a mature area, periodically new discoveries in the design and/or operation renew the interest in distillation, because improvements can translate into very large economic benefits.

Renewed interest in Thermally Coupled Distillation (TCD) appeared in the last 15-20 years, due the important potential savings in energy, with typical values around $10 \%$ to $40 \%$ (in some cases even larger) that have been reported ${ }^{5-10}$ when compared to conventional distillation sequences. Although the interest in TCD is relatively recent the first apparatus that uses TDC concepts was due to Wright in $1947^{11}$, although the theoretical basis of TCD was developed in the sixties by Petlyuk and coworkers ${ }^{12}$. Petlyuk and coworkers noticed that separation sequences using conventional columns (a single feed with two product streams, a condenser, and a reboiler) suffer from an inherent inefficiency produced by the thermodynamic irreversibility during the mixing of streams at the feed, top, and bottom of the column. This remixing is inherent to any separation that involves an intermediate boiling component and can be generalized to an $\mathrm{N}$-component mixture. The theoretical studies developed by Petluyk and coworkers showed that this inefficiency can be improved by removing some heat exchangers and introducing thermal coupling between columns. If a heat exchanger is removed, the liquid reflux (or vapor load) is provided by a new stream that is withdrawn from another column; in this way, it is possible to reduce the energy consumption, and under some circumstances, also the capital costs. (See Figure 1) A fully thermally coupled (FTC) configuration is reached when the entire vapor load is provided by a single reboiler and all the reflux by a single condenser. Halvorsen \& Skogestad ${ }^{13-15}$ proved that the minimum energy consumption for an ideal $\mathrm{N}$ component mixture is always obtained for the FTC configuration.

Despite the reduction in energy consumption, there is a price to be paid when using thermally coupled systems:

(1) The energy must be supplied under the worst conditions, namely at the highest temperature in the reboiler, and removed at the lowest temperature in the condenser, preventing in most cases the use of lower cost utilities such as medium- or low-pressure steam.

(2) When using conventional columns, it is common to constrain the alternatives to sharp separations. For example, in a three-component mixture $(A B C)$, sorted by volatilities, we postulate initially separations $A$ from $B C(A / B C)$ and $A B$ from $C(A B / C)$. However, in FTC distillation, we introduce sloppy separations -an intermediate product is allowed to distribute along the column- and, therefore, we increase the number of column sections. This increase does not imply an increase in the number of columns, but usually an 
increase in the total number of trays. A detailed discussion on the number of column sections needed for a given separation can be found in Agrawal ${ }^{16}$ and in Caballero \& Grossmann. ${ }^{5,6,17}$

(3) In FTC systems the minimum vapor flow is that of the most difficult separation ${ }^{14,15}$ and, therefore, some column sections will have large diameters.

(4) Operation is also more difficult due to the large number of interconnections between the columns.

Therefore, it cannot be concluded that complex configurations are always superior, compared to sequences of simple columns. Instead, the optimum configuration will be dependent on the specific mixture and feed conditions.

Besides the energy savings in TCD, there are also possibilities of important investment savings. First, the reduction in energy consumption is due to a reduction of the liquid and vapor flow rates, and therefore, the lower energy consumption, the smaller the diameters required for the distillation column. Second, an important saving in investment can be obtained by using Divided Wall Columns. A FTC configuration for a three component mixture, like that in Figure 2a, (also known as Petlyuk configuration in honor to F. Petlyuk, who discovered it) formed by two columns, can be rearranged in a single shell using an internal wall (as shown in Figure $2 b$ ). Both configurations in Figure 2, the Petlyuk and the Divided Wall Column (DWC), are thermodynamically equivalent since all the flows, temperatures, compositions are the same in both configurations. In practice there are small differences due to the pressure differences needed to control the external streams in the Petlyuk configurations, but in a preliminary design these differences can be neglected. Detailed discussion about Thermodynamically Equivalent configurations can be found in the works of Caballero \& Grossmann ${ }^{18}$ Agrawal and coworkers ${ }^{19,20}$ or Rong et al ${ }^{21,22}$. In this way the separation of a three component mixture that usually requires two distillation columns, can be performed using a single column with an internal wall. The investment savings are evident since we are only using a single column, reducing the land requirements, the pumps, and piping.. However, the design of TCD systems including DWC involving mixtures with more than three components is not straightforward and it is the objective of this work.

Depending on the number of distillation columns used to separate an $\mathrm{n}$ component mixture into $\mathrm{n}$ product streams, the sequence can be classified as more than $\mathrm{n}-1$ columns configurations, as exactly $n-1$ columns, and less than $n-1$ columns configurations.

For the case of zeotropic mixtures, sequences with exactly $n-1$ columns, named basic configurations by Agrawal ${ }^{23}$,, are characterized by the following three features: (1) Mixtures (or states) with the same components are transferred only once from one distillation column to another; (2) a final product is obtained in a single location of the sequence; (3) the feed stream 
and all the intermediate mixtures are split into exactly two product streams by two columns sections.

Configurations that violate the first two features and obey only the third one produce sequences with more than $\mathrm{n}-1$ columns. These configurations, also referred as non-basic, have higher operating cost than the best basic configurations ${ }^{6,24}$. Non-basic configurations also tend to have higher capital cost due to the additional distillation columns, and therefore non-basic configurations can be removed from the search space.

Configurations that violate the third feature have higher operating cost than the best basic configuration due to increased heat duty, especially for obtaining high purity products. However, the reduced number of columns in some situations could compensate the extra energy consumption. In the literature some of these cases can be found, for example those due to Brugma ${ }^{25}$ or Kaibel ${ }^{26}$, Kim et al ${ }^{27}$ or Errico \& Rong ${ }^{28}$. However, in this paper, we focus only on basic configurations and we will study the case of fewer than $n-1$ columns sequences in a future work.

It is important to make some remarks on previous the features and classification.

(1). Previous classification (sequences with less, exactly and more than $n-1$ columns) is referred to as configurations without internal walls.

(2). Sequences obeying the three distinguishing features can always be arranged into $n-1$ columns, although the total number of separation tasks can be larger. Consider the following example. We want to separate a four component mixture $A B C D$ where the components are sorted by decreasing volatilities ( $A$ is the most volatile component, $D$ the least volatile). Assume we perform the following separation tasks: $A B / B C D$-that means separate $A$ from $C D$ letting the $B$ component to be optimal distributed between distillate and bottoms- $A / B ; B C / C D ; B / C$ and $C / D$. In this example we can identify 5 separation tasks, and this sequence can be performed using 5 distillation columns, but this sequence can be easily arranged in three distillation columns (See Figure 3). In fact it can be rearranged in 16 thermodynamically equivalent configurations using three distillation columns ${ }^{18}$.

(3) A Sequence including divided wall columns, or a single DWC like the one shown in Figure 2 , is a thermodynamically equivalent configuration without walls that uses exactly $n-1$ columns and obeys the three distinguish features. In that sense, although strictly speaking DWC uses less than $\mathrm{n}-1$ columns, it can be considered that DWCs are basic configurations.

As a final any sequence of distillation columns can be rearranged in a single column using vertical partitions and various reboilers and/or condensers. In some situations they can even be stacked using horizontal partitions. A detailed discussion is presented by Agrawal ${ }^{29}$. In this 
work we do not consider those alternatives, but just the 'classical' divided wall columns with at most a single reboiler and a single condenser.

In this paper we propose a mathematical programming approach that takes the form of a MixedInteger Nonlinear Programming Problem (MINLP) or General Disjunctive Programming Problem (GDP) to search for the optimal sequence among all the basic configurations, explicitly including DWCs. In order to accomplish this objective, we first develop a set of logic relationships that can be used in a mathematical programming environment in terms of Boolean (GDP) or binary (MINLP) variables that allow only feasible basic sequences and explicitly include DWCs. We will show that basic configurations do not take into account heat exchangers (reboilers and condensers), but in systems with DWCs these heat exchangers can be explicitly taken into account. Finally, several examples are presented.

\section{Problem Statement.}

The problem addressed in this paper can be stated as follows: Given is a mixture of $M$ components that do not form azeotropes. The objective is to generate the best sequence of distillation columns, including conventional, non conventional and explicitly divided wall columns to completely separate $\mathrm{N}(N \leq M)$ components.

With 'complete separation' we are referring to the sharp separation of those $\mathrm{N}$ key components (i.e. high recovery of any of the $\mathrm{M}$ components). The rest of the components are allowed either to distribute in all the final streams in order to perform the separation with the minimum energy consumption, or if the components are previously classified by groups (i.e C3s, C4s, etc), the separation is performed in such a way that all the components in a group exit from the system all together. In any case, the non-key components do not add complexity to the system, and therefore, without loss of generality we can assume that we have an $\mathrm{N}$ component mixture that must be separated into their $\mathrm{N}$ pure components.

Finally, although we are assuming a final sharp separation between all the key components, this does not exclude some sloppy separations (a component is distributed among distillate and bottoms) in any of the intermediate columns.

\section{Logic Approach to TCD sequences with DWCs}

As pointed out in the introduction, a sequence of distillation columns that includes conventional, thermally coupled, and divided wall columns, must include the space of all the basic configurations (recall that a DWC can be considered as two columns sharing a single shell). The first rule based algorithm for generating the full set of basic configurations was proposed by Agrawal ${ }^{23}$. Following this line, later Ivakpour \& Kasiri ${ }^{30}$ proposed a formulation in which 
distillation configurations are presented mathematically as upper triangular matrices. Independently, Shah \& Agrawal ${ }^{31}$ presented an alternative matrix formulation in which distillation configurations are generated by exploring all possible instances of the presence or absence of transfer streams. This last approach is very efficient for generating the full set of basic configurations. All these rule based approaches have proved to be effective when generating alternatives, but the enumeration of all the alternatives is, except in the case of a reduced number of possibilities, an inefficient strategy. While these approaches can be easily adapted for metaheuristic optimization (i.e genetic algorithms, Particle Swarm Optimization, etc.), it is not clear how to adapt them to a deterministic mathematical programming formulation.

Based on the observations in the seminal paper by Agrawal ${ }^{16}$, Caballero \& Grossmann ${ }^{5,6,17}$ proposed a complete set of logical rules in terms of Boolean variables that implicitly include all the basic column configurations. These logical equations can be transformed into linear equations in terms of binary variables, and integrated in a mathematical programming formulation. The objective in those works was not to generate explicitly all the basic configurations, but to develop a set of logic equations that ensure a strong relaxation when solving the resulting MINLPs that include all the performance equations of the distillation columns, for extracting the optimal configuration without an explicit enumeration of all the alternatives. It is interesting to note that Shah \& Agrawal ${ }^{31}$ proposed a valid set of equations in terms of binary variables that can be integrated in a mathematical programming framework. However, their focus was on checking quickly if a given alternative is a basic one (with excellent performance) and not when those equations are integrated with the model of the columns in a mathematical programming framework (i.e. some of their equations can be obtained from the aggregation of some of the logic relations presented by Caballero \& Grossmann ${ }^{5,6}$, and therefore a worse relaxation is expected)

If we try to synthesize a sequence of distillation columns, the concept of basic configuration is not enough. Consider, for example, the Figures $4 a, 4 b$ and $4 c$. These three sequences of columns correspond to the same basic sequence. The difference between Figures $4 \mathrm{a}$ and $4 \mathrm{~b}$ is that they differ in the existence of the internal heat exchangers. An internal heat exchanger is a condenser or reboiler associated to any internal mixture. In other words, a heat exchanger associated to a stream connecting two different columns. Note that the final heat exchangers, that are associated to final products, are the same in the three configurations of Figure 4 . In general, a given basic configuration can differ in the existence or not of internal heat exchangers, but the heat exchangers associated to final products are the same. A detailed discussion about this point can be found in the work by Caballero \& Grossmann ${ }^{6}$. In the case of Figure $4 \mathrm{c}$ is not so obvious that this configuration corresponds to the same basic configuration. But if we look at the sequence of separation tasks, we observe that the three sequences are performing the same sequence of tasks (see Figure $4 d$ ). Figures $4 b$ and $4 c$ are thermodynamically equivalent. 
Figures $4 \mathrm{a}$ and $4 \mathrm{~b}$ are the same basic configuration, but are not equivalent. One would expect that their behavior (and the total cost) be similar in both configurations, but the different structure of internal heat exchangers can produce important differences; i.e. it is known that given a basic configuration the arrangement in columns with minimum energy consumption is that with the maximum possible thermal coupling (that arrangement without internal heat exchangers). However, this reduced numbers of heat exchangers must provide vapor flow to the entire system, and therefore it is likely that some column sections must be designed with larger diameters. So, the internal structure of heat exchangers is important and must also be optimized. Based on these observations, Caballero \& Grossmann ${ }^{5,6,32}$ proposed a sequential algorithm in which first the search was constrained to basic configurations with the maximum thermal coupling. Then for the best basic configuration obtained in the first step the structure of internal heat exchangers is optimized, and finally the optimal column rearrangement is performed taken into account the thermodynamically equivalent alternatives.

The sequential approach cannot guarantee the global optimal solution, but a good solution. However, DWCs introduce an important reduction in the investment costs, and therefore the search space should not be constrained only to the basic configurations, but to the internal structure of heat exchangers that must be simultaneously optimized. However, differences in thermodynamically equivalent alternatives are expected to be very small, mainly due to operational considerations, and therefore, thermodynamically equivalent configurations should not be included in the search space.

In order to develop a set of logical relationships that allow to generate TCD sequences with DWCs, we use the State Task Network (STN) formalism ${ }^{33,34}$. A state is a stream (or a set of streams) that are characterized by a minimum set of relevant features. These can be qualitative, i.e. composition ( $A B C$ mixture inside some specifications), phase liquid or vapor, or quantitative if necessary. $A$ task is a chemical or physical transformation between adjacent states (i.e. separate $A$ from $B C$ in a mixture $A B C$ ).

In the case of TCD, it is interesting to note that a separation task is performed always by two column sections (a column section is a portion of a distillation column which is not interrupted by entering or exiting streams or heat flows). But these two column sections do not necessarily belong to the same final distillation column ${ }^{18}$.

Using the STN formalism, Caballero \& Grossmann ${ }^{5,6,17}$ presented a set of logical relations between separation tasks that ensure feasible sequences. The first step is then to extend those logical equations to take into account the possibility of including DWCs. For a detailed description of these logical relationships the interested reader is referred to the original works. However, for the sake of clarity an outline of those relationships is included in the appendix.

It is important to note that exists a one to one relationship between the sequence of separation tasks and the states formed by those tasks. Therefore, we can take advantage of this relationship, and express the logical relations to include DWCs in terms of states (instead of 
tasks). Although from a theoretical point of view it is possible to generate a multi-wall column that in the extreme case separates all the components in a single column, nowadays only columns with one wall have been built and operate, so we constrain ourselves to a maximum of one wall in a given column. At the end of the paper we have included a small section with an outline of how to systematically generate multi-wall columns.

A divided wall column is formed by the union of three separation tasks or by four states: a feed state, the two states produced by the first separation task and the intermediate product state. For example, Figure 5 shows the different sequences of states that produce DWCs in a five component mixture.

To generate the logical relations that ensure that all alternative DWC are taken into account, it is necessary first to identify which combinations of states (or tasks) are able to generate a DWC. The next conditions assure that all the DWCs are taken into account:

1. All intermediate states (those that do not include a component with extreme volatility) can form part of a DWC; i.e. according to the definition in previous paragraph, the states $A$ and $E$ cannot be part of the 4 states that define a DWC (See Figure 5).

2. The intermediate product state in the DWC must be produced by two different states. i.e in the Figure 5.(c1) state $C$ is produced by $B C$ and $C D$. In Figure $5 b .(c 2)$, state $C$ is produced by states $A B C$ and $C D E$.

3. Each one of the two states that produce the intermediate product in the DWC must be generated by a single contribution; i.e. in Figure 5.(c1) the two states that produce $\mathrm{C}$ are $B C$ and $C D$. Both $B C$ and $C D$ are generated from a single state: $B C$ from $B C D$ and $C D$ from $B C D$.

4. The state that generates intermediate states in the DWC must be produced by the same state; i.e. in Figure 5.(c1) the two states that produce $C$ are $B C$ and $C D$. These two states are generated from the same state BCD. In Figure 5.c2 the two states that produce $C$ are $A B C$ and $C D E$; both, $A B C$ and $C D E$ are produced from $A B C D E$.

With the 4 previous rules, it is straightforward to generate all the possible DWC. For example, in a 5 component mixture there are 15 DWCs ( 3 with $B$ as intermediate product; 4 with $C$ as intermediate product; 3 with D; 2 with BC; 2 with $C D$ and 1 with BCD. See Figure 5)

In order to explicitly include DWC in a GDP or MINLP model, it is necessary to add some logical relationships that ensure the feasibility of the sequence. Therefore, to the set of logical rules previously developed by Caballero \& Grossmann ${ }^{5,6,17}$-See appendix- we must add the logical relationships shown in next paragraphs.

First let us define the following index sets: 
$D W C=\{w \mid w$ is a DWC $\}$; i.e. In a five component mixture one out of the 15 possibilities commented above.

STATES $=\{s \mid s$ is a state; i.e. $A, B, C \ldots A B, B C, C D \ldots A B C, B C D$, etc

$D W Y_{w, s}=\{$ States $s$ that form part of the DWC $w\}$

$\mathrm{DWN}_{\mathrm{w}, \mathrm{s}}=\{$ States that cannot appear simultaneously to the DWC $\mathrm{w}\}$

$I \mathrm{NC}_{\mathrm{w}, \mathrm{s}}=\{$ Set of DWCs $\mathrm{w}$ sharing the state $\mathrm{s}\}$

$\mathrm{DWINT}_{\mathrm{w}, \mathrm{s}}=\{$ internal states $\mathrm{s}$ that form part of the DWC $\mathrm{w}\}$

1.- The first logical relationship simple relates the DWCs with the states:

$$
\begin{array}{ll}
C W_{w} \Rightarrow Z_{s} & \forall w, s \in D W Y_{w, s} \\
C W_{w} \Rightarrow \neg Z_{s} & \forall w, s \in D W N_{w, s}
\end{array}
$$

where the Boolen variable $\mathrm{CW}_{\mathrm{w}}$ takes the value true if the DWC $\mathrm{w}$ exists, and False otherwise. And the Boolean variable $Z$ is True if the state s exists and zero otherwise.

2.- If two or more DWCs share a state, at most one of those columns can be selected.

at most one ${ }_{w \in I N C_{w, s}}\left(D W_{w}\right) \forall s\left(\sum_{w \in I N C_{w, s}} d w_{w} \leq 1 \forall s\right)$

For example, among the following configurations: $\mathrm{B} 1 ; \mathrm{C} 1 ; \mathrm{C} 4 ; \mathrm{BC} 1 ; \mathrm{BC} 2$-referred to Figure 5 - that share the state $\mathrm{BC}$ we can select at most one.

3.- Any heat exchanger associated to an internal state in a DWC must not be selected

$$
C W_{w} \Rightarrow \underset{s \in D W C I N T_{s, w}}{\wedge}\left(\neg W_{s}\right)
$$

where the Boolean variable Ws makes reference to a heat exchanger associated to the state s.

One of the characteristics of this logic based approach is it flexibility for including any other constraints. For example, it is of interest not to allow thermally coupled between the DWC and the rest of the system. In other words, we can force to the DWCs to have reboiler and condenser, and maybe to force the feed to the DWCs to be a single stream.

To do this, we add the following index set and constraint:

$\mathrm{DWCOND}_{\mathrm{w}, \mathrm{s}}=\{$ State $\mathrm{s}$ that form part of the DWC $\mathrm{w}$ and could have a condenser $\}$

DWCOND $_{\mathrm{w}, \mathrm{s}}=\{$ State $\mathrm{s}$ that form part of the DWC $\mathrm{w}$ and could have a reboiler $\}$ 


$$
C W_{w} \Rightarrow W C_{s} \wedge W R_{s s} \quad s \in D W C O N D_{w, s} s s \in D W R E B_{w, s s} \forall w \in D W C
$$

\section{Model}

In this section we present a comprehensive description of the model. The first step is to generate a superstructure that includes all the alternatives. In this case, as indicated above, we will follow the STN formalism ${ }^{34}$. Generating the superstructure is straightforward: we only need to generate all possible separation tasks and all the states and join the tasks with the states that the task produces and vice versa. However, the visualization of this superstructure is not easy. Fortunately, there is a one to one relationship between the sequence of states and the sequence of separation tasks. Therefore, we will use only the states, only the tasks or both to get the maximum clarity. (See Figure 6).

A Separation task is formed by two column sections that are named, by similarity with conventional columns, rectifying and stripping sections. However, in the final configuration of the actual columns these two sections are not necessarily placed in the same column. In that sense we can consider a task as a pseudo-column. (Figure 7 helps to clarify this point and shows some important variables used in the model).

In this work we use the Underwood, Gilliland, Fenske equations ${ }^{35,36}$ to calculate the minimum vapor flowrate (and minimum liquid flowrate) in each pseudo-column (task). We use the following heuristics that at the same time simplify the problem:

a. The reflux ratio is assumed to be 1.2 times the minimum.

b. Each separation is performed assuming a fixed recovery of key components -typically greater than 0.95-. In this way it is possible to estimate a priori the minimum number of separation trays. According to the Fenske equation, the minimum number of trays is given by:

$N_{\min }=\frac{\operatorname{Ln}\left[\left(\frac{x_{i}}{x_{j}}\right)_{D}\left(\frac{x_{j}}{x_{i}}\right)_{B}\right]}{\operatorname{Ln}\left(\alpha_{i, j}\right)}$

where $\mathrm{i}$ and $\mathrm{j}$ make reference to the light key and heavy key components, respectively. Subscript $D$ and $B$ make reference to the distillate and bottoms streams respectively, and $\alpha$ is the relative volatility of the light key respect to the heavy key.

If we write previous equation in terms of molar flow rates of key components, and taking into account that:

$$
\begin{aligned}
& f_{i}=d_{i}+b_{i} ; \quad d_{i}=\varepsilon_{i} f_{i} ; \quad b_{i}=\left(1-\varepsilon_{i}\right) f_{i} \text { i is the light key } \\
& f_{j}=d_{j}+b_{j} ; \quad d_{i}=\left(1-\varepsilon_{j}\right) f_{j} ; \quad b_{j}=\varepsilon_{j} f_{j} \quad j \text { is the heavy key }
\end{aligned}
$$


equation (5) can be written as follows:

$$
N_{\min }=\frac{\operatorname{Ln}\left[\left(\frac{x_{i}}{x_{j}}\right)_{D}\left(\frac{x_{j}}{x_{i}}\right)_{B}\right]}{\operatorname{Ln}\left(\alpha_{i, j}\right)}=\frac{\operatorname{Ln}\left[\left(\frac{d_{i}}{d_{j}}\right)\left(\frac{b_{j}}{b_{i}}\right)\right]}{\operatorname{Ln}\left(\alpha_{i, j}\right)}=\frac{\operatorname{Ln}\left[\left(\frac{\varepsilon_{i}}{1-\varepsilon_{i}}\right)\left(\frac{\varepsilon_{j}}{1-\varepsilon_{j}}\right)\right]}{\operatorname{Ln}\left(\alpha_{i, j}\right)}
$$

c. The actual number of trays can then be calculated using the Gilliland correlation:

$$
\frac{N_{a c t}-N_{\min }}{N_{a c t}+1}=0.75\left[1-\left(\frac{R R-R R_{\min }}{R R+1}\right)^{0.5688}\right]
$$

where $R R$ is the reflux ratio. Using the heuristic that $R R=1.2 \cdot R R_{\min }$, the right hand side of the equation varies between 0.6 and 0.5 for minimum reflux ratios between 0.5 and 5 . In this way, we do not introduce too much error if we assume a constant value around 0.53. Of course the equation can be included in the model, but this avoids including a nonlinear and non-convex equation.

In order to develop the model the following index sets must be defined:

1. $\operatorname{TASK}=\{\mathrm{t} \mid \mathrm{t}$ is a given task $\}$

e.g. TASK $=\{(A B C / B C D),(A B / B C D),(A B C / C D),(A B / B C),(A B / C)$ $(B / C D),(B C / C D),(A / B),(B / C),(C / D)\}$

2. STATES $=\{\mathrm{s} \mid \mathrm{s}$ is a state $\}$ e.g. STATES= $\{(A B C D),(A B C),(B C D),(A B),(B C),(C D)$,

$$
(A),(B),(C),(D)\}
$$

3. $\mathrm{IM}_{\mathrm{S}}=\{\mathrm{S} \mid \mathrm{s}$ is an intermediate state. All but initial and final products $\}$ e.g. IMS $=\{(A B C),(B C D),(A B),(B C),(C D)\}$

4. $\operatorname{COMP}=\{\mathrm{i} \mid \mathrm{i}$ is a component to be separated in the mixture $\}$ e.g. $C O M P=\{A, B, C, D\}$

5. $\mathrm{FS}_{\mathrm{T}}=\{\mathrm{t} \mid \mathrm{t}$ is a possible initial task; Task that receives the external feed $\}$ e.g. TASK $\{(A / B C D),(A B / B C D),(A B / C D),(A B C / B C D),(A B C / C D),(A B C / D)\}$

6. $\mathrm{TS}_{\mathrm{S}}=\{$ tasks $\mathrm{t}$ that the state $\mathrm{s}$ is able to produce $\}$

e.g. $\quad \mathrm{TS}_{\mathrm{ABCD}}=\quad\{(\mathrm{AB} / \mathrm{BCD}),(\mathrm{ABC} / \mathrm{BCD}),(\mathrm{ABC} / \mathrm{CD})\}$ 


$$
\begin{array}{ll}
\mathrm{TS}_{\mathrm{ABC}}= & \{(\mathrm{AB} / \mathrm{BC}),(\mathrm{AB} / \mathrm{C})\} \\
\mathrm{TS}_{\mathrm{BCD}}= & \{(\mathrm{B} / \mathrm{CD}),(\mathrm{BC} / \mathrm{CD})\}
\end{array}
$$

7. $\mathrm{ST}_{\mathrm{S}}=\{$ tasks $\mathrm{t}$ that are able to produce state $\mathrm{s}\}$

$$
\begin{aligned}
& \text { e.g } \quad \mathrm{ST}_{\mathrm{ABC}}=\quad\{(\mathrm{ABC} / \mathrm{CD}),(\mathrm{ABC} / \mathrm{BCD})\} \\
& S T_{B C D}=\quad\{(A B / B C D),(A B C / B C D)\} \\
& S T_{A B}=\quad\{(A B / B C D),(A B / B C),(A B / C)\} \\
& \mathrm{ST}_{\mathrm{BC}}=\quad\{(\mathrm{AB} / \mathrm{BC}),(\mathrm{BC} / \mathrm{CD})\} \\
& \mathrm{ST}_{\mathrm{CD}}=\quad\{(\mathrm{ABC} / \mathrm{CD}),(\mathrm{B} / \mathrm{CD}),(\mathrm{BC} / \mathrm{CD})\}
\end{aligned}
$$

8. $R E C T s=\{t a s k t$ that produces state $s$ by a rectifying section $\}$

e.g. $\quad R E C T_{A B C}=\{(A B C / C D),(A B C / B C D)\}$

$$
\begin{array}{ll}
\operatorname{RECT}_{\mathrm{AB}}= & \{(\mathrm{AB} / \mathrm{BCD}),(\mathrm{AB} / \mathrm{BC}),(\mathrm{AB} / \mathrm{C})\} \\
\mathrm{RECT}_{\mathrm{BC}}= & \{(\mathrm{BC} / \mathrm{CD})\}
\end{array}
$$

9. STRIPs $=\{$ task $t$ that produces state $s$ by a stripping section $\}$

e.g. $\quad$ STRIP $_{B C D}=\{(A B / B C D),(A B C / B C D)\}$

$$
\begin{array}{ll}
\text { STRIP }_{B C}= & \{(A B / B C)\} \\
\text { STRIP }_{C D}= & \{(A B C / C D),(B / C D),(B C / C D)\}
\end{array}
$$

10. $\mathrm{FPs}=\{\mathrm{s} \mid \mathrm{s}$ is a final state (pure products) $\}$

e.g. $\quad F P=\{(A),(B),(C),(D)\}$ do not confuse with components, although the name is the same.

11. $P R E_{i}=\{$ tasks $t$ that produce pure product $i$ through a rectifying section $\}$

$$
\begin{array}{lll}
\text { e.g. } & \mathrm{PRE}_{\mathrm{A}}= & \{(\mathrm{A} / \mathrm{B})\} \\
& & \\
\mathrm{PRE}_{\mathrm{B}} & =\{(\mathrm{B} / \mathrm{CD}),(\mathrm{B} / \mathrm{C})\} \\
& \mathrm{PRE}_{\mathrm{C}}= & \{(\mathrm{C} / \mathrm{D})\}
\end{array}
$$

12. $\mathrm{PST}_{\mathrm{i}}=\{$ tasks $\mathrm{t}$ that produce pure product $\mathrm{i}$ through a stripping section $\}$

$$
\begin{array}{rll}
\text { e.g. } & \mathrm{PST}_{\mathrm{B}}= & \{(\mathrm{A} / \mathrm{B})\} \\
& \mathrm{PST}_{\mathrm{C}}= & \{(\mathrm{AB} / \mathrm{C}),(\mathrm{B} / \mathrm{C})\}
\end{array}
$$




$$
\mathrm{PST}_{\mathrm{D}}=\quad\{(\mathrm{C} / \mathrm{D})\}
$$

13. $U R=\{r \mid r$ is Underwood root $\}$

$$
\text { e.g. }\{r 1, r 2, r 3, r 4\}
$$

14. $A U R_{t}=\{r$ is an active Underwood root in task $t\}$

e.g. $\quad A \cup R_{A / B C D}=\{r 1\}$

$A \cup R_{A B / B C D}=\{r 1, r 2\}$

$A U R_{A B C / B C D}=\{r 1, r 2, r 3\}$

$A \cup R_{A B / C D}=\{r 2\}$ etc.

We introduce the following continuous variables:

$F_{t} \quad$ Feed molar flow that enters in the separation task $\mathrm{t}$

$D_{t} \quad$ Molar flow that exits from rectifying section of task $\mathrm{t}$

$B_{t} \quad$ Molar flow that exits from the stripping section of task $\mathrm{t}$

$f_{t, i} \quad$ Molar flow of component $\mathrm{i}$ the enters in the separation task $\mathrm{t}$

$d_{t, i} \quad$ Molar flow of component $\mathrm{i}$ that exits from rectifying section of task $\mathrm{t}$

$b_{t, i} \quad$ Molar flow of component $\mathrm{i}$ that exits from the stripping section of task $\mathrm{t}$

$L 1_{t} \quad$ Liquid molar flow in rectifying section of task $\mathrm{t}$

$L 2_{t} \quad$ Liquid molar flow in stripping section of task $\mathrm{t}$

$V 1_{t} \quad$ Vapor molar flow in rectifying section of task $\mathrm{t}$

$V 2_{t} \quad$ Vapor molar flow in stripping section of task $\mathrm{t}$

Ps Molar flow of a stream that leaves the system (final product)

QCond $_{t} \quad$ Heat load in the condenser in task $\mathrm{t}$

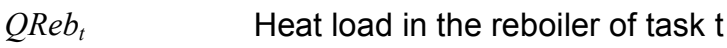

and the following Boolean variables.

$Y_{t} \quad$ True if the separation task $\mathrm{t}$ exists. False otherwise.

Zs $\quad$ True if the state s exists. False, otherwise

Ws True if the heat exchanger associated to the state s exists. False, otherwise

The objective of the model is to minimize the total annualized cost. Therefore, the objective function is as follows:

$$
\min : \mathrm{TAC}=\frac{r(1+r)^{L}}{(1+r)^{L}-1}[\text { Capital costs }]+\text { Operating costs }
$$


where the capital cost are annualized assuming a depreciation interval of $L=8$ years in this work and an interest rate ( $r=0.09$ in this work -9\%-). This corresponds to a capital charge factor of 0.18 ; i.e. $18 \%$ of the capital costs are charged annually to cover interest on spent capital and depreciation.

The rest of the model includes the following equations:

1. Disjunctions related with the existence or not of a given separation task:

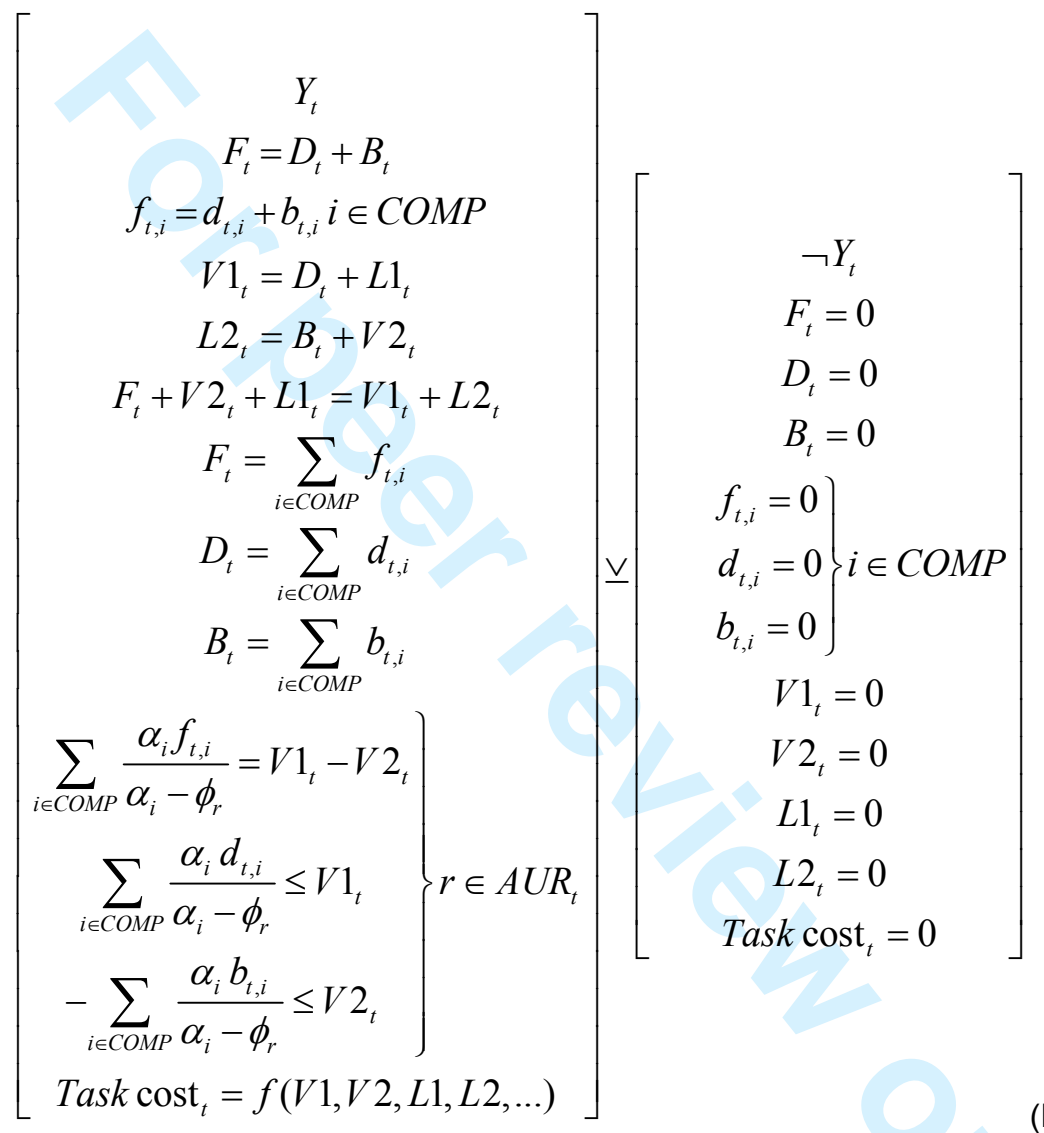

The second term of the disjunction ensures that if a task does not exist all the flows and costs are forced to be zero. Note also that the Underwood root $\left(\phi_{r}\right)$ is left free in that case.

The first five equations in the first term of the disjunction are, respectively, the total mass balance in the task, component mass balance in the task, mass balances in condenser, reboiler and feed tray. The next three equations are Underwood equations and the last is to calculate the task cost.

Related to the Underwood equations there are several important remarks to take into account: 
The second and third Underwood equations are written as inequalities. The reason is that the original Underwood equations calculate the minimum reflux -or minimum internal vapor flows-. But in a Thermally Coupled system it is possible that some columns cannot operate at minimum (or 1.2 times the minimum) due to the vapor (and liquid) transfer between columns. Therefore the 'dominant' column will force the other to increase the vapor and liquid flows. Figure 8 clarifies this point

In a TCD system is not correct to assume that the feed to a given column is liquid at its bubble point. In fact, if we connect the rectifying section of a task with another task through a thermal couple, these two streams are equivalent to a single superheated vapor stream whose flow is the net flow. If we connect the stripping section of a task with another task through a thermal couple these two streams are equivalent to a subcooled liquid stream (a detailed discussion can be found in the works by Carlberg \& Westerberg ${ }^{37,38}$ and Navarro et al ${ }^{39}$ ). This fact is explicitly taken into account in the model because we maintain the two streams (liquid and vapor) that form a thermal couple, instead of calculating the equivalent thermal state of the feed. Usually the right hand side of the first Underwood equation is written in terms of the feed thermal state as follows:

$$
\sum_{i \in C O M P} \frac{\alpha_{i} f_{i}}{\alpha_{i}-\phi_{r}}=F(1-q)
$$

But a simple mass balance in the feed tray (See Figure7) allows removing the variable $q$ (liquid fraction), and at the same time to remove a nonlinear and nonconvex term:

$$
\begin{aligned}
& V 1=F_{V}+V 2 \\
& L 2=F_{L}+L 1
\end{aligned}
$$

the liquid fraction is defined as:

$$
q=\frac{F_{L}}{F}=\frac{L 2-L 1}{F} ; \quad \text { or } \quad 1-q=\frac{V 1-V 2}{F}
$$

and therefore,

$$
F(1-q)=V 1-V 2
$$

It is worth noting also that the previous disjunction does not take into account the existence or not of heat exchangers (reboiler and/or condenser). In other words, the task requires reflux and a vapor load streams, but for the disjunction it is not relevant where those streams come from (i.e. from a heat exchanger of from a thermal couple). 


\section{Connectivity equations and equations related to the existence or not of a given state.}

Connectivity equations are flow relations between tasks. They can be modeled by admitting that a state generates a mixer and a splitter. The connectivity equations depend also on the existence or not of a heat exchanger. If a heat exchanger does not exist, the connectivity between two tasks must also take into account the transfer of vapor and liquid streams that form a thermal couple.

2.1. Mass balance in the feed to the system:

$$
\begin{aligned}
& F_{0}=\sum_{t \in F S_{t}} F_{t} \\
& f_{0 i}=\sum_{t \in F S_{t}} f_{t, i}
\end{aligned}
$$

\subsection{Intermediate states: Mass and energy balances and cost calculations}

In disjunction $D 2$, the variable $Z_{\mathrm{s}}$ takes the value true if the state $s$ exists and false otherwise. It is important to recall that there is a one to relationship between a sequence of tasks and a sequence of states. Therefore, the introduction of the new boolean variables $\mathrm{Zs}$ do not increase the combinatorial complexity of the model. Even more, if the problem is solved using a MINLP reformulation, it is necessary to define as binary variables those that are either related to tasks, or those that are related to states (the other can be defined as a continuous variables bounded between 0 and 1). The logical relationships will force the other set of variables to take the correct integer values. (See appendix).

The second term in the main disjunction (when the boolean Zs takes the value of false), is introduced for the sake of completeness, but it is redundant. Note that if a given state does not exists, the logical relationships will force that all the task that could be generated by the state, and all the tasks that could generate the state, do not exist as well. Therefore, the second term in disjunction D-1 also forces the variables related with those tasks to be all zero.

The disjunction inside the first term in D-2 is related to the existence or not of a heat exchanger in a state (if Ws is True heat exchanger is selected). Again, it is worth noting that there is a one to one relationship between assigning heat exchangers to states or to tasks. The logical relationships force that if one is selected (i.e. tasks), the corresponding correct state is selected and vice versa. If the heat exchanger is selected, the equations are different depending if the heat exchanger is a condenser or a reboiler. The inner most disjunction (those related to WCs -True if heat exchanger is a condenser- or WRs -True if heat exchanger is a reboiler-) includes the energy balance in condenser or reboiler and the cost equations. 
If the heat exchanger does not exist $\left(\neg W_{s}\right)$, but the state exists, then we have a thermal couple. Equations inside this term of the disjunction are simply mass balances to ensure the correct liquid and vapor flow transfer between columns.

Figure 9a, 9b shows a conceptual graphical description of disjunction D-2 that helps to understand the meaning of each term. 


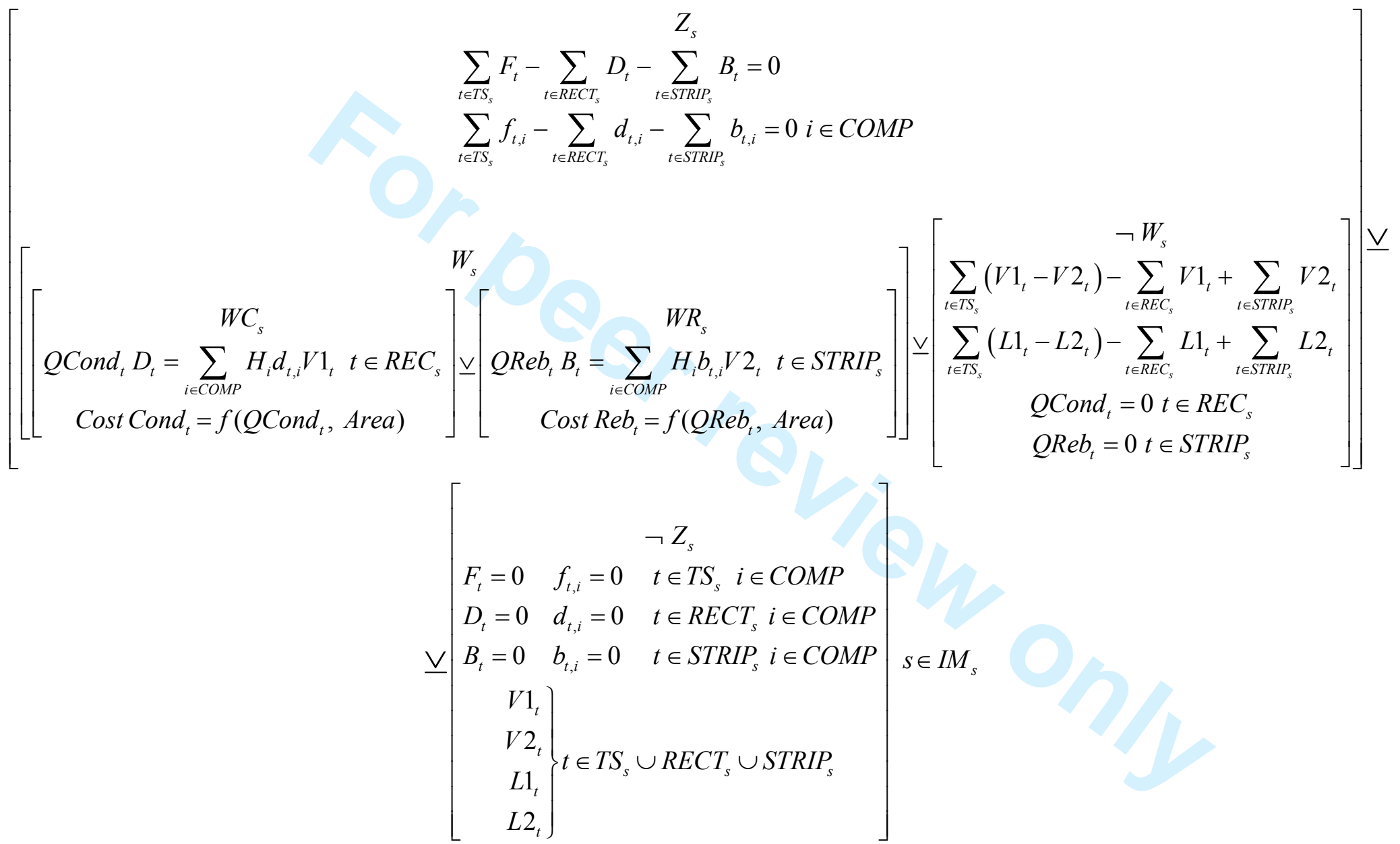




\subsection{Final states. Mass and energy balances and cost calculations.}

A final state is a state related with a pure product, or in general with a stream that leaves the system (the sequence of columns), and then these states must always exist. The most volatile product will always have a condenser and the heaviest a reboiler. However, the rest could have a condenser or a reboiler if it is produced by a single contribution, or no heat exchanger at all if produced by two contributions. In this last case the internal liquid and vapor flows of at least one of the tasks that generate the state must be adjusted to satisfy the mass balances (see Figure 8). The following disjunction shows this situation. Figure 10 shows a conceptual representation of disjunction D-3.

$$
\left.\left[\begin{array}{c}
W R_{s} \\
\text { cost Reb }= \\
f(\text { Qreb, Area })
\end{array}\right] \vee\left[\begin{array}{c}
W C_{s} \\
\text { cost Cond }= \\
f(\text { Qcond, Area })
\end{array}\right]\right]\left[\begin{array}{c}
\neg W_{s} \\
\sum_{t \in P R E_{s}} V 2_{t}=\sum_{t \in P S T_{s}} V 1_{t} \\
\sum_{t \in P R E_{s}} L 2_{t}=\sum_{t \in P S T_{s}} L 1_{t}+P_{s}
\end{array}\right] \forall s \in F P_{s}
$$

\subsection{Recovery of final products}

$$
\begin{aligned}
& \sum_{t \in P R E_{s}} d_{i, t}+\sum_{t \in P S E_{s}} b_{i, t} \geq r e c_{i} f_{0, i} \quad \forall i \in C O M P, s \in F P \\
& \sum_{t \in P R E_{s}} D_{t}+\sum_{t \in P S E_{s}} B_{t}=P_{s} \quad s \in F P
\end{aligned}
$$

where 'rec' is the global recovery of each component typically between 0.95 and 0.999 . In this way we ensure the final desired sharp separation of each component.

\section{Existence of a Divided Wall Column}

As discussed previously, a divided wall column is thermodynamically equivalent to a set of three fully thermally coupled tasks (no internal heat exchangers). The presence or not of a condenser and a reboiler depends on the designer preferences or maybe to control considerations, but they are not strictly necessary. In any case, the only difference in the initial design is in the cost of the column. Note that any extra consideration related with the controllability, or even to the designer preferences, can easily be added through logical relationships or extra constraints.

The cost of a distillation column can be estimated by adding the costs of the trays (or the packing), the cost of the vessel and the costs of heat exchangers (reboilers and 
condensers). Note that the existence or not of a heat exchanger does not depend on the existence of a task. Therefore, we can separate the vessels from the heat exchangers. It is worth remembering that a separation task can be considered as a pseudo-column, so we can calculate the costs of trays as the addition of the costs of each task. If we want that the number of total trays in the sections that share a wall be the same in the two sides of the wall, the simplest possibility is to assume that the most restrictive situation is the dominant one. In other words, we can assume that the total number of trays in the zone of the internal wall is equal to the maximum trays in each part of the wall.

A different situation appears when we want to calculate the cost of the vessels that form the distillation columns. In general, consider a separation task as a column, and simply adding the costs of all the vessels, is not correct because the number of tasks can be greater than the number of actual columns. As mentioned above, independently of the number of tasks that form a sequence, all basic sequences can be arranged in $\mathrm{N}-1$ columns. If we include the DWCs, the number of vessels decreases by one for each DWC introduced. The major difficulty in calculating the cost of the vessel is that we should know which column sections form a given column, but this is precisely something that we want to avoid because a given sequence of tasks could be rearranged in a large number of thermodynamically equivalent configurations.

A simple approach to solve this problem consists of taking advantage of the fact that according to Turton et al ${ }^{40}$ the purchased cost of a vertical vessel varies linearly with its volume. See Figure 11

$C_{p}=C_{f}+C_{v} \mathrm{Vol}$

where $\mathrm{Vol}$ is the vessel volume. Therefore, we can add the volume of all the vessels, and taking into account that in a basic configuration there are $(\mathrm{N}-1)$ vessels minus the number of divided wall columns the total cost of the vessels can be calculated as follows:

$$
\text { Total Vessels Cost }=(N-1) C_{f}-\sum_{w \in D W C} C_{f} C W_{w}+\sum_{t \in T a s k} C v_{t} V_{o l}
$$

Before going into the examples, there are some interesting points about the proposed model.

The disjunctive model is reformulated as a mixed integer non-linear programming (MINLP) problem, using a big-M formulation ${ }^{41,42}$ and written in GAMS ${ }^{43}$. The problem can be solved using any standard solver. However, the non-convexities and the large number of local solutions in this type of problem often lead to suboptimal solutions. However, based on the following observations it is possible solve the model and get a very good solution, in most of the cases, the global optimal solution. 
a. Problems with a fixed topology (fixed binary variables) produce the optimal solution and rarely get trapped in local solutions. The same can be said for the resulting nonlinear problem (NLP) with a relaxation of the binary variables (we use the solver CONOPT ${ }^{44}$ under GAMS). This fact can be checked comparing the results of the local optimizer with a deterministic global solver like BARON ${ }^{45}$.

b. For a fixed sequence of separation tasks (or states), the optimal solution tends to be a solution with a large degree of thermal couples in the internal states. In other words, the sequence without internal heat exchangers is likely to be near the optimal solution for that configuration. Remember that an internal heat exchanger is a heat exchanger associated to a stream -state- that connects two separation tasks. Heat exchangers associated to a final product (a stream that leaves the system) are fixed with the sequence of tasks.

Based on observation (a) a simple branch and bound based solver, that solves only NLPs in a tree search, like SBB ${ }^{46}$ in GAMS, is expected to find a near global optimal solution, which in many cases we expect to be the global optimal solution. Note that although BARON is able to solve each of the nodes to global optimality, the CPU time is orders of magnitude larger than the CPU time used by a local solver, and therefore, from a computational point of view, using a global NLP deterministic optimizer inside the branch and bound search is prohibitive.

Decomposition techniques like the Outer Approximation ${ }^{47,48}$ or the Generalized Benders Decomposition ${ }^{49}$ are another alternative. However, in this case the Master problem is very sensitive to the nonconvexities, cutting off important parts of the feasible region. As a result we often get trapped in suboptimal solutions.

Therefore in this work we solve the model using SBB. We sacrifice some computational performance in order to obtain a good solution.

Based on observation (b), it is also possible to develop a strategy that in most cases get a solution close to the best solution in a fraction of the CPU time. The algorithm is as follows:

1. Fix the value of all internal heat exchangers to False (zero).

2. Using branch and bound solve the resulting MINLP. Note that DWCs are allowed if we relax the condition that a divided wall column must have a reboiler and a condenser.

3. For the solution in step 2, fix the sequence of tasks and determine the optimal distribution of internal heat exchangers. The solution of step 3 is an upper bound to the optimal solution of the problem.

4. Repeat from step 1 until in two consecutive iterations there is no improvement in the upper bound. 
Of course, previous algorithm is based on a heuristics rule but numerical test have proved that usually get a solution very close to the optimal.

\section{Example 1. Separation of a mixture of aromatic Hydrocarbons}

This example illustrates some of the capabilities of the proposed model. Here we present the separation of a five component mixture (Benzene, Toluene, EthylBenzene, Styrene, alpha methyl styrene). All the relevant data for the problem is presented in Table 1. According to the previous comments in the paper, we constrain the search to basic configurations: the sequence of tasks and states can be rearranged in $\mathrm{N}-1$ actual distillation columns. Each DWC reduces by one the number of actual columns.

First, we solve the model allowing all the thermal couples. i.e. DWCs can be thermally coupled with the rest of the system. The best solution obtained (sol 1.1) is shown in Figure 12, with a TAC of $\$ 946,130$ /year. The optimal solution includes a DWC formed by the following tasks $(B C / C D ; B / C, C / D)$. The arrangement in actual columns shown in Figure $12 b$ is only one out of the four thermodynamically equivalent configurations that maintain the existence of the DWC. The solution obtained has two important issues related with the DWC. First, the areas in both sides of the DWC are very different, and second, the areas in the same side but different sections of the DWC are also very different. Although it is possible to build DWCs in which the wall is not centered, if the deviation is too large hydrodynamic problems makes the DWC an invalid option. This drawback can be easily solved adding a constraint that forces DWCs to have similar areas in both sides of the wall (i.e. a maximum deviation around 20-30\%). If we force the feed and the intermediate product to be saturated liquid, the vapor flow rate in different sections, but in the same side of the wall will be equal and then the areas will also be similar. Including these constraints, the solution is the same in terms of states and tasks but without including DWCs. The total annual costs increases to $\$ 950,150 /$ year. (sol 1.2)

In this first case, because the dominant cost is the energy, the optimal solution is almost a fully thermally coupled configuration. It is remarkable, however, the presence of a heat exchanger (a condenser) in the task $A B / B C D$. Removing that heat exchanger and introducing a thermal link instead is likely to produce some decrease in the total energy consumption. However, the thermal links also produce an increase in the total vapor flow through the entire system increasing the diameters of some columns.

The cost correlations, the constant volatilities assumption, and some of the rules of thumb introduce some uncertainty in the model. Therefore, it is of interest to obtain not only the best solution but also the set of solutions inside a given tolerance (i.e. $5 \%$ ). This set of solutions should be evaluated with detail using rigorous models. As an example, the Figure 13 shows the second best solution (sol 1.3) with a TAC of $\$ 951,500$ / year that is only $0.5 \%$ more expensive than the best configuration. In this case the optimal solution includes a DWC. 
In both solutions the DWCs are extensively thermally coupled and integrated with the rest of the system. It is also of interest to force the DWCs to be not thermally coupled with the rest of the system (the DWC must have a condenser a reboiler). This can be done by adding logical constraints (eq. 5). If we do that, and force the existence of at least a DWC, the optimal solution is shown in Figure 14 (sol 1.4). The TAC increases to $\$ 1,004,453 /$ year (6 \% more expensive).

All previous models take between $20-30$ min of CPU time to solve (using SBB under GAMS version windows 7, 64 bit in a machine with a processor Intel Core2 Quad 2.40GHz and 8GB of RAM). If instead of using a branch and bound search over the original model, we use the heuristic algorithm proposed in previous section (maintaining the constraints to assure feasible walls) we obtain a solution involving a DWC with a TAC $\$ 952,270$ / year (sol 1.5) -See Figure 15- Just a $0.08 \%$ larger than the best solution in only $3.7 \mathrm{~min}$ of CPU time.

It should be noted that there are a relatively large number of alternatives with similar TAC, but important structural differences, which adds an extra degree of freedom that can be used to select a good alternative taking into account other considerations (controllability, environmental considerations, etc.)

The potential of thermally coupled distillation is clear if we compare it to the optimal solution using conventional columns (each column is forced to have a condenser and a reboiler) and sharp separation in each column -classical column sequencing-. The optimal configuration in this case is shown in Figure 16, (sol 1.6) with a TAC $\$ 1,528,174$ / year which is $\underline{61.5 \% \text { more }}$ expensive!

Finally, in order to establish the numerical performance or the model and solution algorithms Table 2 shows some statistics about the models in this and next examples.

\section{Example 2. Separation of a mixture of Linear Hydrocarbons,}

In this example we separate a mixture of 5 linear hydrocarbons (from n-hexane to $n$-decane) in which the relative volatilities between two consecutive components is similar, and the separations are easier than in the previous example. Table 3 shows all the relevant data of this problem.

In this case the optimal solution has a TAC of $\$ 632,258 /$ year (sol 2.1) and includes the maximum number of column sections ${ }^{16}[\mathrm{~N}(\mathrm{~N}+1)]$. Also it is almost a fully thermally coupled configuration with only an internal condenser in state $A B$ and an internal reboiler in state $B C D E$, (see Figure 17). In this example, the flows between internal states with two contributions (BCD, $B C, C D$ ) have been forced to be saturated liquid. Although from a theoretical point of view, we can save some energy allowing thermal couples in this situation; this could produce important control problems and column section with very different diameters. Constraints mentioned in the first example about feasibility in DWCs are maintained. In this case, the optimal solution does not include a DWC showing that DWCs are not always the best alternative. In some situations 
the DWCs are not even feasible. But one interesting conclusion is that it is likely that there are solutions not involving DWCs with similar performance.

If instead of solving the full model we use the two stage algorithm, the optimal solution increases to a TAC of $\$ 646,381$ year (sol 2.2) that is only a $2.2 \%$ larger than the best obtained solution (see Figure 18), confirming that usually thermally coupled systems have a large number of solutions with similar TAC, which provides the designer with an extra degree of freedom to select the best configuration taking into account any other extra considerations.

Again, if we consider the sequence formed only by conventional columns, the best sequence has a TAC of $\$ 1,119,697 /$ year (sol 2.3 ) that is $77 \%$ more expensive than the best solution. (Figure 19)

\section{Extension to multiwall column systems}

Although, to our knowledge only columns with a single wall have been built and satisfactorily operated, it is theoretically possible to extend the methodology to multiwall columns. It is not the objective of this paper to present a comprehensive study of multiwall columns. However, a general outline of how to deal with the conceptual design of these systems can be of interest.

Let us first focus on systems with four components. In this case, a single column with two walls should be able to separate the four components. Following the philosophy of considering only basic configurations, we must study only the space of fully thermally coupled configurations (a single reboiler and a single condenser in all the system). According to Agrawal ${ }^{16}$ or Caballero \& Grossmann ${ }^{17}$ the number of separations tasks in FTC systems varies between $(4 N-6) / 2$ and $N(N-1) / 2$ (6 separation tasks and 5 separation task respectively in the 4 components case). The sequence of tasks and a possible arrangement of the walls for the sequence with the maximum number of column sections are shown in Figure 20a, but it is not clear is how to deal with the two directional flows (mixture BC in Figure 20a). The problem is produced by the two contributions that form the state BC. Therefore, it would be desirable to constrain the search to fully thermally coupled systems in which all the states are formed by a single contribution. Fortunately, all the sequences with $(4 N-6) / 2$ separation tasks fulfill this requirement. Figures $20 \mathrm{~b}$ to $20 \mathrm{~d}$ show all the FTC configurations with $(4 N-6) / 2$ separation tasks. Among these sequences only sequences $20 \mathrm{c}$ and $20 \mathrm{~d}$ can be rearranged in a two wall column (Figure 21). One interesting observation is that starting with a sequence of $(4 N-6) / 2$ tasks, the two wall configurations can be obtained by first searching for alternatives with a single wall, then group the states that form that DWC in a single state, and repeat the procedure, see Figure 21. This recursive approach can be extended to sequences with more than four components to locate 
alternatives with two or more walls. As an example, consider Figure 22, in which a three wall column is generated from a sequence of $(4 \cdot 5-6) / 2=7$ separation tasks.

Finally it is important to point out that although is not difficult to generate all the alternatives that use one, two or more walls for a given $\mathrm{N}$-component mixture, the feasibility of these alternatives depends on factors such as the hydrodynamics (i.e. walls give rise to different transversal areas in a given sections), controllability (distribution of vapor and liquid flows in the different sides of the walls) or mechanical (i.e. construction of the trays or packed distribution in each section or sections with very different transversal areas). The geometrical distribution of trays could also be important (i.e. Figure 22 shows at least two alternatives for the wall distribution inside a column for a 5 component mixture)

\section{Conclusions and final remarks}

This paper has introduced a methodology for the design of distillation columns sequences of zeotropic mixtures explicitly including divided wall columns. Some remarkable characteristics of the proposed approach are the following:

1. The model is very flexible, and includes from sharp conventional sequences (each column has a reboiler and a condenser -classical column sequencing) to fully thermally couple configurations (a single reboiler and a single condenser for all the sequence of columns), going through all the intermediate possibilities, and explicitly including DWCs.

2. Constraining the search to any of the extreme cases presented in the previous paragraphs, or including any preference of the designer is very straightforward by just including or removing some logical constraints in the model.

3. The search is constrained to basic configurations. A DWC can be included if we take into account that a DWC can be considered thermodynamically equivalent to a fully thermally coupled subsystem formed by three separation tasks. However, the particularities of the DWCs make necessary the explicit identification of these substructures. Again, the model proved to be very flexible and logical relationships easily control the possibility of decoupling or not (through heat exchangers) the DWC from the rest of the sequence.

4. In this work we presented a shortcut model approach based on the Underwood-Fenske equations. However, the disjunctive representation of the model accommodates any other aggregated or even rigorous model. In any case, the approach presented here is enough for the preliminary design. It allows identifying some promising alternatives for a latter detailed simulation.

5. When we consider thermally coupled systems with DWCs, it is common that there are a good number of alternatives with similar economic performance, but the best solution not necessarily includes a DWC. Although these alternatives increases the difficulty of 
the system, at the same time it provides an extra degree of freedom to the designer that could take into account other considerations (i.e. controllability, environmental considerations, safety, etc) in the final design.

6. Even though the DWCs are explicitly taken into account, the model is based on a tasks approach instead of a column approach. The reason is that when a thermal couple appears, it introduces two thermodynamic equivalent configurations. In a preliminary design all these thermodynamic equivalent alternatives must be avoided in order to avoid a large redundancy (i.e. large number of equivalent configurations with the same performance from the total annual cost point of view).

The extension to multiwall systems, if not straightforward, is not difficult. The major difficulty is in identifying structures that are able of being rearranged in a multiwall column. An outline of how to, recursively, identify these alternatives was also presented. However, in this case other considerations (hydrodynamics, controllability etc) are not yet to our knowledge, satisfactorily solved.

A final remark could be of interest. Any sequence of distillation columns can be rearranged in a single column using vertical partitions and various reboilers and/or condensers. In some situations they can even stacked using horizontal partitions. A detailed discussion was presented by Agrawal ${ }^{29}$. In this work we do not consider those alternatives but just the 'classical' divided wall columns with at most a single reboiler and a single condenser.

\section{ACKOWLEDGMENTS}

The authors wish to acknowledge support from the Spanish Ministry of Science and Innovation (CTQ2009-14420-C02).

\section{APPENDIX}

Here we present the logical relationships to ensure a feasible basic column sequence. Besides the index sets included in the text we must include the following.

$P_{-} R_{E C}=\{$ task $t$ that produces final product $s$ through a rectifying section. $\}$

$\mathrm{P}_{-} \mathrm{STR}_{\mathrm{S}}=\{$ task $\mathrm{t}$ that produces final product $\mathrm{s}$ through a stripping section. $\}$

The rest of index sets are variables were defined in the text.

1. A given state $s$ can give rise to at most one task.

$$
\underset{t \in T S_{t}}{\bigvee} Y_{t} \vee K ; s \in C O L
$$


where $\mathrm{K}$ is a dummy boolean variable that means "do not choose any of the previous options".

2. A given state can be produced at most by two tasks: one must come from the rectifying section of a task and the other from the stripping section of a task

$$
\left.\begin{array}{l}
\underset{t \in R E C T_{S}}{\bigvee} Y_{t} \vee K \\
\underset{t \in \text { STRIPSS }}{\vee} Y_{t} \vee K
\end{array}\right\} s \in \text { STATES }
$$

where $\mathrm{K}$ has the same meaning than in equation (1). Note that if we want only systems with the minimum number of column sections at a given state, except products, it should be produced at most by one contribution. Note also that when at least a state is produced by two contributions, the number of separation tasks is not the minimum.

3. All the products must be produced at least by one task.

$$
\underset{t \in\left(P_{-} R E C_{s} \cup P_{-} S T R_{s}\right)}{\vee} Y_{t} ; s \in F P
$$

4. If a given final product stream is produced only by one task, the heat exchanger associated with this state (product stream) must be selected.

$$
\left.\begin{array}{l}
\neg\left(\underset{t \in P_{-} R E C_{s}}{\bigvee_{t}} Y_{t}\right) \Rightarrow W_{s} \\
\neg\left(\bigvee_{t \in P_{-} S T R_{s}}^{\bigvee_{t}} Y_{t}\right) \Rightarrow W_{s}
\end{array}\right\} \quad s \in F P
$$

5. If a given state is produced by two tasks (a contribution coming from a rectifying section and the other from a stripping section of a task) then there is not a heat exchanger associated to that state (stream).

$$
\left(Y_{t} \wedge Y_{k}\right) \Rightarrow \neg W_{s} \quad\left\{\begin{array}{l}
t \in R_{E C T} \\
k \in \operatorname{STRIP}_{s} \\
s \in \text { STATES }
\end{array}\right.
$$

6. Connectivity relationships between tasks in the superstructure

$$
\left.\begin{array}{l}
Y_{t} \Rightarrow \bigvee_{k \in T S_{s}} Y_{k} ; \quad t \in S T_{s} \\
Y_{t} \Rightarrow \bigvee_{k \in S T_{s}} Y_{k} ; \quad t \in T S_{s}
\end{array}\right\} s \in \text { STATES }
$$

7. If a heat exchanger associated to any state is selected then a task which generates that state must also be selected.

$W_{s} \Rightarrow \bigvee_{S T_{s}} Y_{t} ; \quad s \in$ STATES 
8. If a separation task t produces a state $s$ by a rectifying section, and that state has a heat exchanger associated, then it must be a condenser. If the state is produced by a stripping section then it must be a reboiler.

$Y_{t} \wedge W_{s} \Rightarrow W C_{s} \quad t \in R E C T_{s}$

$Y_{t} \wedge W_{s} \Rightarrow W R_{s} \quad t \in \operatorname{STRIP}_{S}$

It is convenient to complete the pervious rule adding that:

9. If a given state does not have a heat exchanger, then both WC and WR associated to that state must be False.

$\neg W_{S} \Rightarrow \neg W C_{S} \wedge \neg W R_{S} \quad s \in S T A T E S$

It is important to note that if the problem is solved as an MI(N)LP or GDP the variables wc and wr do not need to be declared as binary and they can be considered as continuous with values between 0 and 1. Previous equations (A8,A9) force wc and wr to take integer values as far as $y$ and $w$ are integer. Therefore, variables wr and wc do not increase the combinatorial complexity of the problem.

10. It is worth mentioning that the set of logical rules previously presented in terms of separation tasks could be easily rewritten in terms only of states: "There is a one to one correspondence between the sequence of tasks and the sequence of states and viceversa". The relationship between tasks and states is as follows:

$Y_{t} \Rightarrow Z_{s} ; \quad t \in S T_{S}$

$Z_{s} \Rightarrow \bigvee_{t \in T S_{s}} Y_{t}$

Equation (A10) could be read as: "if the task $t$, that belongs to the set of task produced by the state $s$, exits then the state s must exist". And equation (A11) as: "If the state $s$ exists at least one of the tasks that the state $s$ is able to produce must exist"

We should note that if the problem is solved as an $\mathrm{MI}(\mathrm{N}) \mathrm{LP}$, it is only necessary declare as binary either yt or zs, but not both. Whether yt is declared as binary zs can be declared as continuous between zero and one and vice-versa.

\section{REFERENCES}

1. Humphrey J. Separation processes: playing a critical role. Chemical Engineering Progress. 1995;91(10):43-54.

2. Mix $T$, Dweck J, Weinberg $M$, Armstrong $R$. Energy conservation in distillation. Chemical Engineering Progress. 1978;74(4). 
3. Soave G, Feliu JA. Saving energy in distillation towers by feed splitting. Applied Thermal Engineering. 2002;22(8):889.

4. Kunesh J, Kister H, Lockett M, Fair JR. Distillation: still towering over other options. Chemical engineering Progress. 1995;91(10).

5. Caballero JA, Grossmann IE. Design of distillation sequences: from conventional to fully thermally coupled distillation systems. Computers \& Chemical Engineering. Oct 15 2004;28(11):2307-2329.

6. Caballero JA, Grossmann IE. Structural considerations and modeling in the synthesis of heat-integrated-thermally coupled distillation sequences. Industrial \& Engineering Chemistry Research. Dec 6 2006;45(25):8454-8474.

7. Fidkowski ZT. Distillation configurations and their energy requirements. AlChE Journal. Jun 2006;52(6):2098-2106.

8. Fidkowski ZT, Agrawal R. Multicomponent thermally coupled systems of distillation columns at minimum reflux. AIChE Journal. Dec 2001;47(12):2713-2724.

9. Rudd H. Thermal Coupling For Energy Efficiency. Chemical Engineer-London. Aug 27 1992(525):S14-S15.

10. Triantafyllou C, Smith R. The Design And Optimization Of Fully Thermally Coupled Distillation-Columns. Chemical Engineering Research \& Design. Mar 1992;70(2):118132.

11. Wright RO, Inventor. Fractionation Apparatus. US patent 24711341949.

12. Petlyuk FB, Platonov VM, Slavinsk.Dm. Thermodynamically Optimal Method For Separating Multicomponent Mixtures. International Chemical Engineering. 1965;5(3):555-\&.

13. Halvorsen IJ, Skogestad S. Minimum energy consumption in multicomponent distillation. 1. V-min diagram for a two-product column. Industrial \& Engineering Chemistry Research. Feb 5 2003;42(3):596-604.

14. Halvorsen IJ, Skogestad S. Minimum energy consumption in multicomponent distillation. 2. Three-product Petlyuk arrangements. Industrial \& Engineering Chemistry Research. Feb 5 2003;42(3):605-615.

15. Halvorsen IJ, Skogestad S. Minimum energy consumption in multicomponent distillation. 3. More than three products and generalized Petlyuk arrangements. Industrial \& Engineering Chemistry Research. Feb 5 2003;42(3):616-629.

16. Agrawal R. Synthesis of distillation column configurations for a multicomponent separation. Industrial \& Engineering Chemistry Research. Apr 1996;35(4):1059-1071.

17. Caballero JA, Grossmann IE. Generalized disjunctive programming model for the optimal synthesis of thermally linked distillation columns. Industrial \& Engineering Chemistry Research. May 16 2001;40(10):2260-2274.

18. Caballero JA, Grossmann IE. Thermodynamically equivalent configurations for thermally coupled distillation. AIChE Journal. Nov 2003;49(11):2864-2884.

19. Agrawal R. More operable fully thermally coupled distribution column configurations for multicomponent distillation. Chemical Engineering Research \& Design. Sep 1999;77(A6):543-553.

20. Agrawal R, Fidkowski ZT. More operable arrangements of fully thermally coupled distillation columns. AlChE Journal. Nov 1998;44(11):2565-2568. 
21. Rong BG, Kraslawski A, Turunen I. Synthesis and optimal design of thermodynamically equivalent thermally coupled distillation systems. Industrial \& Engineering Chemistry Research. Sep 1 2004;43(18):5904-5915.

22. Rong BG, Turunen I. A new method for synthesis of thermodynamically equivalent structures for Petlyuk arrangements. Chemical Engineering Research \& Design. Dec 2006;84(A12):1095-1116.

23. Agrawal R. Synthesis of multicomponent distillation column configurations. AIChE Journal. Feb 2003;49(2):379-401.

24. Giridhar A, Agrawal R. Synthesis of distillation configurations: I. Characteristics of a good search space. Computers \& Chemical Engineering. 2010;34(1):73.

25. Brugma AJ. Process and Device for Fractional Distillation of Liquid Mixtures. U.S. Patent 2.295.256. 1942.

26. Kaibel G. Distillation Columns with Vertical Partitions. Chemical Engineering Techology. 1987;10:92.

27. Kim JK, Wankat PC. Quaternary distillation systems with less than N-1 columns. Industrial \& Engineering Chemistry Research. Jul 7 2004;43(14):3838-3846.

28. Errico M, Rong B-G. Modified simple column configurations for quaternary distillations. Computers \&amp; Chemical Engineering. 2012;36(0):160-173.

29. Agrawal R. Multicomponent distillation columns with partitions and multiple reboilers and condensers. Industrial \& Engineering Chemistry Research. Oct 3 $2001 ; 40(20): 4258-4266$.

30. Ivakpour J, Kasiri N. Synthesis of Distillation Column Sequences for Nonsharp Separations. Industrial \& Engineering Chemistry Research. 2009;48(18):8635-8649.

31. Shah VH, Agrawal R. A matrix method for multicomponent distillation sequences. Aiche Journal. 2010;56(7):1759-1775.

32. Caballero JA, Grossmann IE. Logic Based Methods for Generating and Optimizing Thermally Coupled Distillation Systems. Paper presented at: European Symposium on Computer Aided Process Engineering-122002; The Hague, The Netherlands.

33. Kondili E, Pantelides CC, Sargent RWH. A General Algorithm For Short-Term Scheduling Of Batch-Operations .1. Milp Formulation. Computers \& Chemical Engineering. Feb 1993;17(2):211-227.

34. Yeomans H, Grossmann IE. A systematic modeling framework of superstructure optimization in process synthesis. Computers \& Chemical Engineering. Jun 1 1999;23(6):709-731.

35. King CJ. Separation Processes. New York: McGraw-Hill; 1980.

36. Underwood A. Fractional distillation of multicomponent mixtures. Chemical Engineering Progress. 1948;44:603-614.

37. Carlberg NA, Westerberg AW. Temperature Heat Diagrams For Complex Columns .2. Underwoods Method For Side Strippers And Enrichers. Industrial \& Engineering Chemistry Research. Sep 1989;28(9):1379-1386.

38. Carlberg NA, Westerberg AW. Temperature Heat Diagrams For Complex Columns .3. Underwoods Method For The Petlyuk Configuration. Industrial \& Engineering Chemistry Research. Sep 1989;28(9):1386-1397. 
39. Navarro MA, Javaloyes J, Caballero JA, Grossmann IE. Strategies for the robust simulation of thermally coupled distillation sequences. Computers \&amp; Chemical Engineering. 2012;36(0):149-159.

40. Turton R, Bailei RC, Whiting WB, Shaeiwitz JA. Analysis Synthesis and Design of Chemical Processes. New York: McGraw-Hill.; 2003.

41. Grossmann IE. Review of Nonlinear Mixed-Integer and Disjunctive Programming Techniques. Optimization and Engineering. 2002(3):227-252.

42. Vecchietti A, Lee S, Grossmann IE. Modeling of discrete/continuous optimization problems: characterization and formulation of disjunctions and their relaxations. Computers \& Chemical Engineering. Mar 15 2003;27(3):433-448.

43. Rosenthal RE. GAMS - A User's Guide: GAMS Development Corporation, Washington, DC, USA; 2012.

44. Drud AS. CONOPT: A System for Large Scale Nonlinear Optimization. Reference Manual. Bagsvaerd, Denmark: ARKI Consulting and Development A/S; 1996.

45. Sahinidis NV. BARON: A general purpose global optimization software package. Journal Of Global Optimization. Mar 1996;8(2):201-205.

46. Bussieck MR, Drud AS. A new solver for mixed integer nonlinear programming. In Recent Advances in Nonlinear Mixed Integer Optimization. INFORMS. Invited talk. 2000.

47. Duran MA, Grossmann IE. A Mixed-Integer Nonlinear-Programming Algorithm For Process Systems Synthesis. AIChE Journal. Apr 1986;32(4):592-606.

48. Yuan X, Pibouleau L, Domenech S. Experiments in process synthesis via Mixed Integer Programming. Chemical Engineering Process. 1989;25(2):99-116.

49. Geofrion AM. Generalized Benders Decomposition. Journal of Optimization Theory and Applications. 1972;10(4):237-259. 
Table 1. Data for example 1.

\begin{tabular}{lcccc}
\hline \multicolumn{1}{c}{ Component } & $\begin{array}{c}\text { Feed composition } \\
\text { (mol fraction) }\end{array}$ & $\alpha_{i, j}{ }^{*}$ & $\begin{array}{c}\Delta \mathrm{H}_{\text {vap }} \\
(\mathbf{k J} / \mathbf{m o l})\end{array}$ & MW \\
\hline (A)- Benzene & 0.3 & 10.5 & 30.77 & 78 \\
(B)- Toluene & 0.2 & 4.04 & 33.19 & 92 \\
(C)- Ethylbencene & 0.1 & 1.76 & 35.58 & 106 \\
(D)- Styrene & 0.2 & 1.31 & 36.83 & 104 \\
(E)- $\alpha$-methyl & 0.2 & 1 & 38.30 & 118 \\
styrene & & & \\
& & & \\
Total Flow & $200 \mathrm{kmol} / \mathrm{h}$ & & \\
Pressure & $1 \mathrm{~atm}$ \\
Steam cost & $5.00 \$ / \mathrm{GJ}(8000 \mathrm{~h} /$ year of operation & \\
Cold water cost & $0.19 \$ / \mathrm{GJ}$ \\
Recovery & 0.98 of light and heavy key in each separation & \\
\hline
\end{tabular}

${ }^{*}$ Relative volatility of component $i$, with respect to the heaviest one (a-methyl styrene (component $j$ )). 
Table 3. Data for example 2

\begin{tabular}{|c|c|c|c|c|}
\hline Component & $\begin{array}{l}\text { Feed composition } \\
\text { (mol fraction) }\end{array}$ & $\alpha_{i, j}{ }^{*}$ & $\begin{array}{c}\Delta \mathrm{H}_{\text {vap }} \\
(\mathrm{kJ} / \mathrm{mol})\end{array}$ & MW \\
\hline (A)- n-hexane & 0.1 & 17.5 & 29.00 & 86 \\
\hline (B)- n-heptane & 0.2 & 8.37 & 31.90 & 100 \\
\hline (C)- n-octane & 0.3 & 4.04 & 34.75 & 114 \\
\hline (D)- n-nonane & 0.3 & 1.99 & 37.29 & 128 \\
\hline (E)- n-decane & 0.1 & 1 & 39.63 & 142 \\
\hline Total Flow & \multicolumn{4}{|l|}{$200 \mathrm{kmol} / \mathrm{h}$} \\
\hline Pressure & \multicolumn{4}{|l|}{$1 \mathrm{~atm}$} \\
\hline Steam cost & \multicolumn{4}{|c|}{$5.00 \$ / G J(8000 \mathrm{~h} /$ year of operation } \\
\hline Cold water cost & \multicolumn{4}{|l|}{0.19 \$/GJ } \\
\hline Recovery & \multicolumn{4}{|c|}{0.98 of light and heavy key in each separation } \\
\hline
\end{tabular}

${ }^{*}$ Relative volatility of component $i$, with respect to the heaviest one (n-decane $j$ )). 
Table 4. Results and model statistics in example 2.

\begin{tabular}{|c|c|c|c|c|c|c|c|}
\hline & \multicolumn{7}{|c|}{ Model } \\
\hline & \multicolumn{3}{|c|}{ Sol 2.1} & \multicolumn{2}{|c|}{ Sol 2.2} & \multicolumn{2}{|c|}{ Sol 2.3} \\
\hline Total vessels cost $(k \$)$ & \multicolumn{3}{|c|}{413.994} & \multicolumn{2}{|c|}{468.127} & \multicolumn{2}{|c|}{361.006} \\
\hline Total tray cost $(k \$)$ & \multicolumn{3}{|c|}{123.810} & \multicolumn{2}{|c|}{135.268} & \multicolumn{2}{|c|}{96.383} \\
\hline Total reboilers cost $(k \$)$ & \multicolumn{3}{|c|}{244.198} & \multicolumn{2}{|c|}{192.704} & \multicolumn{2}{|c|}{477.674} \\
\hline Total condensers Cost $(k \$)$ & \multicolumn{3}{|c|}{215.735} & \multicolumn{2}{|c|}{266.341} & \multicolumn{2}{|c|}{438.680} \\
\hline Total steam cost ( $k \$ /$ year) & \multicolumn{3}{|c|}{439.376} & \multicolumn{2}{|c|}{442.061} & \multicolumn{2}{|c|}{844.981} \\
\hline Total cooling water cost ( $k \$ /$ year) & \multicolumn{3}{|c|}{13.290} & \multicolumn{2}{|c|}{13.081} & \multicolumn{2}{|c|}{27.442} \\
\hline Total Investment $(k \$)$ & \multicolumn{3}{|c|}{997.737} & \multicolumn{2}{|c|}{1062.440} & \multicolumn{2}{|c|}{1373.743} \\
\hline TAC (charging factor $=0.18)(\mathrm{k} \$$ /year $)$ & \multicolumn{3}{|c|}{632.258} & \multicolumn{2}{|c|}{646.381} & \multicolumn{2}{|c|}{1119.697} \\
\hline Sequence of separation tasks * & $\begin{array}{l}\mathrm{ABCD} / \mathrm{B} \\
\mathrm{ABC} / \mathrm{BC} \\
\mathrm{BCD} / \mathrm{CD} \\
\mathrm{AB} / \mathrm{BC}^{\mathrm{C}} \\
\mathrm{BC} / \mathrm{CD} \\
\mathrm{CD} / \mathrm{DE} \\
\mathrm{A} / \mathrm{B}^{\mathrm{C}} \\
\mathrm{B} / \mathrm{C} \\
\mathrm{C} / \mathrm{D} \\
\mathrm{D} / \mathrm{E}_{\mathrm{R}} \\
\end{array}$ & $\begin{array}{l}3 C D E_{R} \\
C D \\
D E \\
\end{array}$ & $\begin{array}{l}(6) \\
(8) \\
(8) \\
(11) \\
(11) \\
(12) \\
(22) \\
(22) \\
(22) \\
(23) \\
\end{array}$ & $\begin{array}{l}A B C D / B C D E \\
A B C / B C D \\
A / B C^{C} \\
B C / C D \\
C D / D E \\
B / C^{C} \\
C / D \\
D / E_{R}\end{array}$ & $\begin{array}{l}(6) \\
(8) \\
(22) \\
(11) \\
(12) \\
(22) \\
(22) \\
(23)\end{array}$ & $\begin{array}{l}\mathrm{AB} / \mathrm{CDE}^{\mathrm{C}}{ }_{\mathrm{R}} \\
\mathrm{C} / \mathrm{DE}^{\mathrm{C}}{ }_{\mathrm{R}} \\
\mathrm{A} / \mathrm{B}^{\mathrm{C}}{ }_{\mathrm{R}} \\
\mathrm{D} / \mathrm{E}^{\mathrm{C}}{ }_{\mathrm{R}}\end{array}$ & $\begin{array}{l}(22) \\
(22) \\
(22) \\
(23)\end{array}$ \\
\hline$D W C s$ (states in the DWC) & \multicolumn{3}{|c|}{ No } & \multicolumn{2}{|c|}{$\begin{array}{c}\text { ABCDE- ABCD- } \\
\text { BCDE }\end{array}$} & \multicolumn{2}{|c|}{ No } \\
\hline CPU time $(s)^{++}$ & \multicolumn{3}{|c|}{1673.7} & \multicolumn{2}{|l|}{225.1} & \multicolumn{2}{|c|}{2.4} \\
\hline$N^{\circ}$ equations & \multicolumn{3}{|c|}{3043} & \multicolumn{2}{|c|}{$3043(2933)^{* *}$} & \multicolumn{2}{|c|}{1189} \\
\hline$N^{\circ}$ continuous variables & & 1347 & & $1338(131$ & & 66 & \\
\hline Binary variables & & 65 & & $56(30$ & & 2 & \\
\hline
\end{tabular}

* Super-index $C$ indicates a condenser in the task; sub-index $R$ indicates a reboiler in the task. Number in parenthesis indicates the number of actual trays

${ }^{++}$Using SBB under GAMS in Windows 7; Processor Intel(R) Core(TM)2Q Quad CPU 2.40GHz 2.39 GHz; RAM 8.00GB

${ }^{* *}$ Numbers out of the parenthesis correspond to the outer problem. Numbers inside the parenthesis correspond to inner problem. 
Sequence of conventional columns (left) and thermal couple (right) by removing the condenser in the first column. $254 \times 190 \mathrm{~mm}(300 \times 300$ DPI $)$ 


1
2
3
4
5
6
7
8
9
10
11
12
13
14
15
16
17
18
19
20
21
22
23
24
25
26
27
28
29
30
31
32
33
34
35
36
37
38
39
40
41
42
43
44
45
46
47
48
49
50
51
52
53
54
55
56
57
58
60

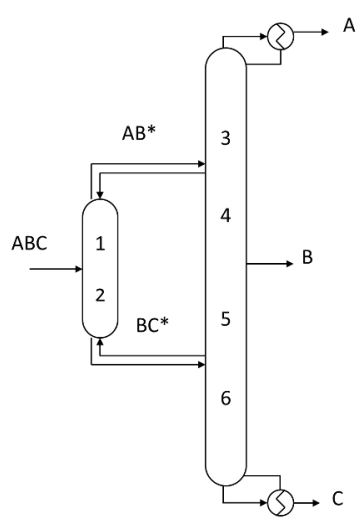

(a)

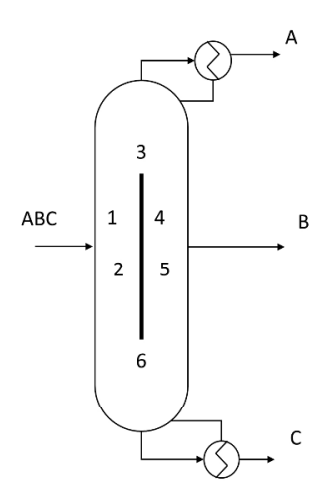

(b)

(a) Petlyuk configuration. (b) Divided Wall Column. Both are thermodynamically equivalent. $254 \times 190 \mathrm{~mm}(300 \times 300 \mathrm{DPI})$ 


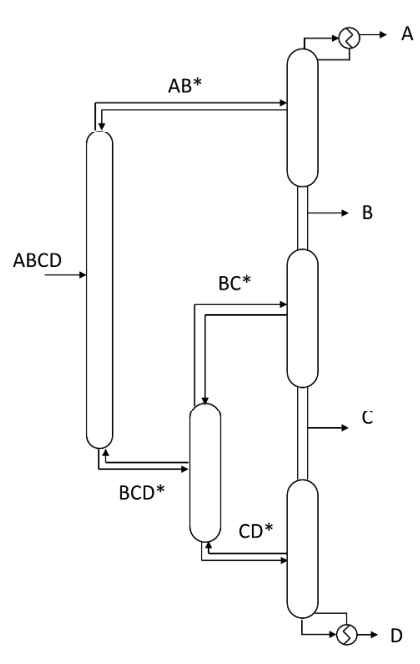

(a)

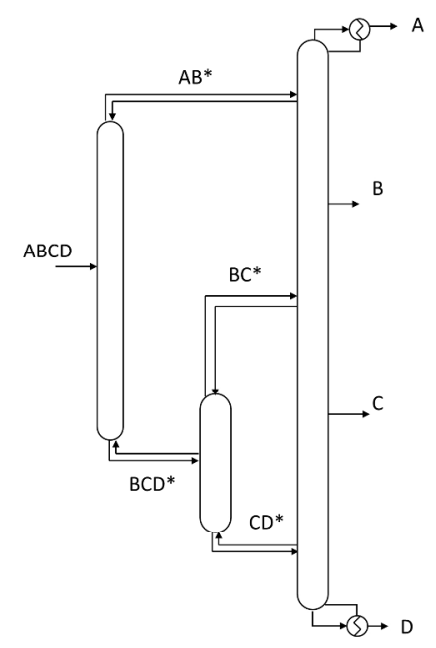

(b)

(a) Separation sequence using 5 columns and (b) one out of the 16 possibilities rearrangement using three distillation columns. $254 \times 190 \mathrm{~mm}(300 \times 300$ DPI $)$ 

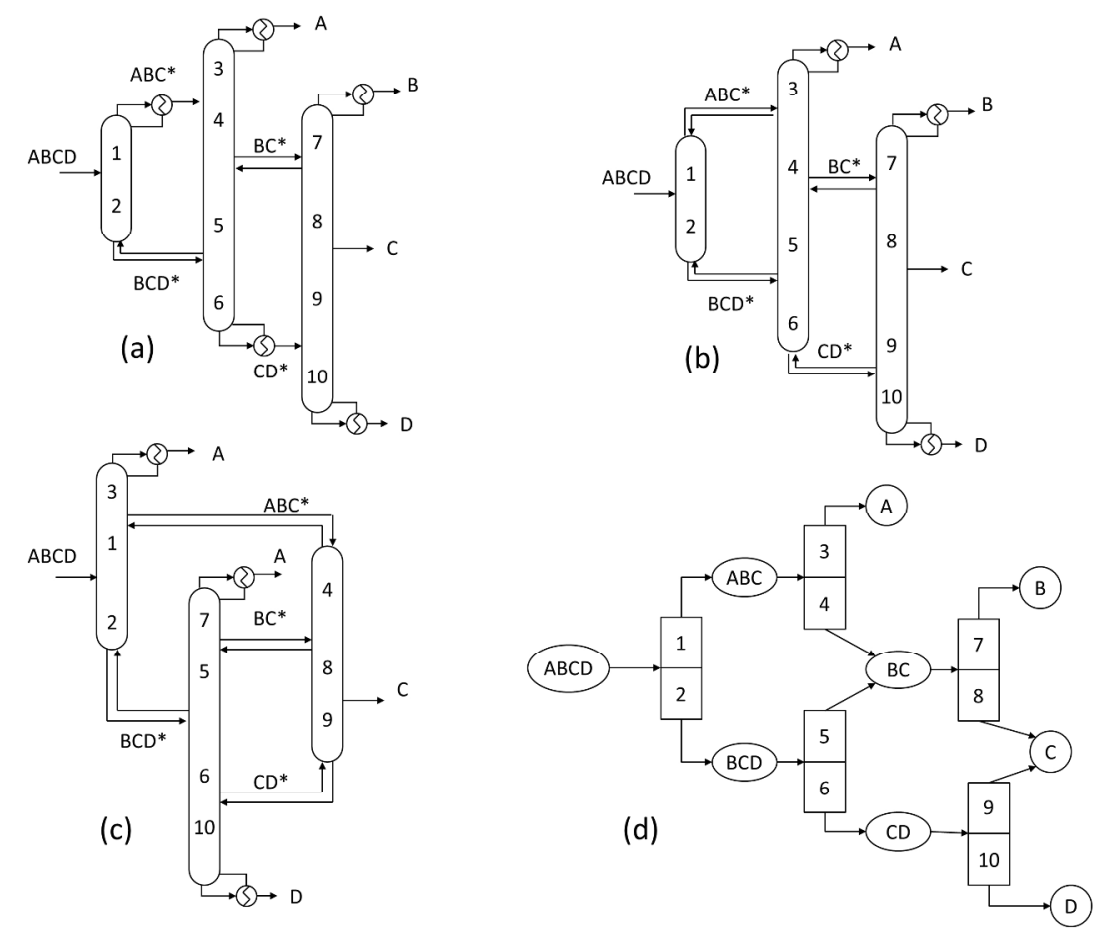

Three sequences of columns with the same basic sequence. (a) and (b) differ in the internal heat exchangers and they are not equivalent. Figures (b) and (c) are thermodynamically equivalents. (d) Sequence of separation tasks common to the three column arrangements $254 \times 190 \mathrm{~mm}(300 \times 300 \mathrm{DPI})$ 

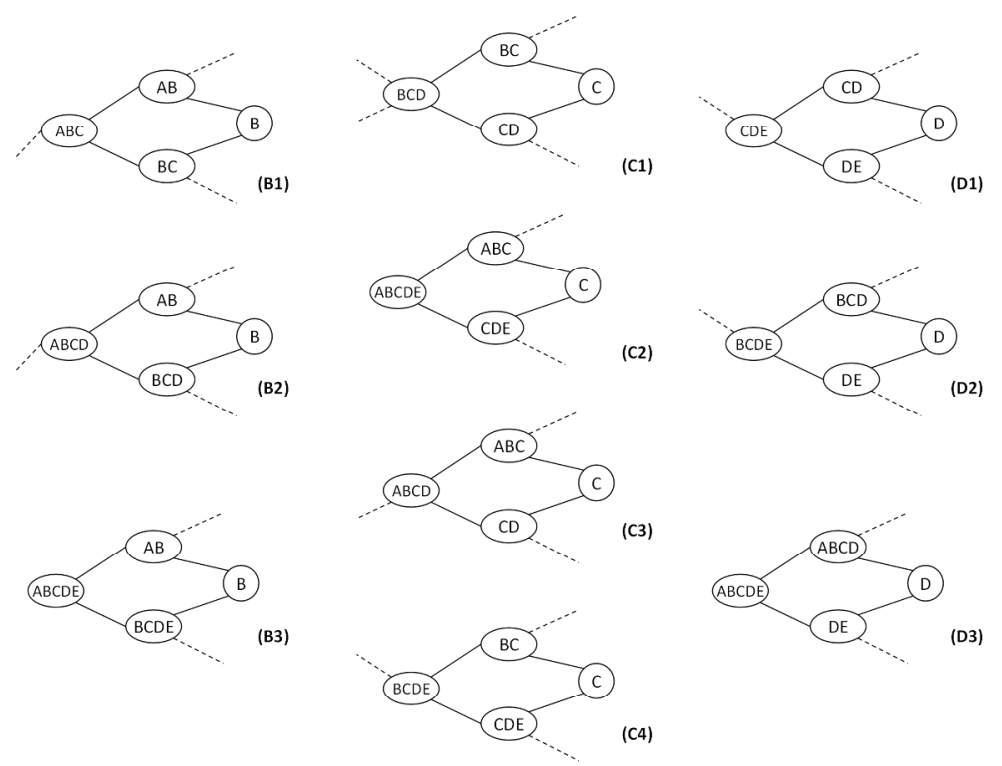

(D3)

$(4)$
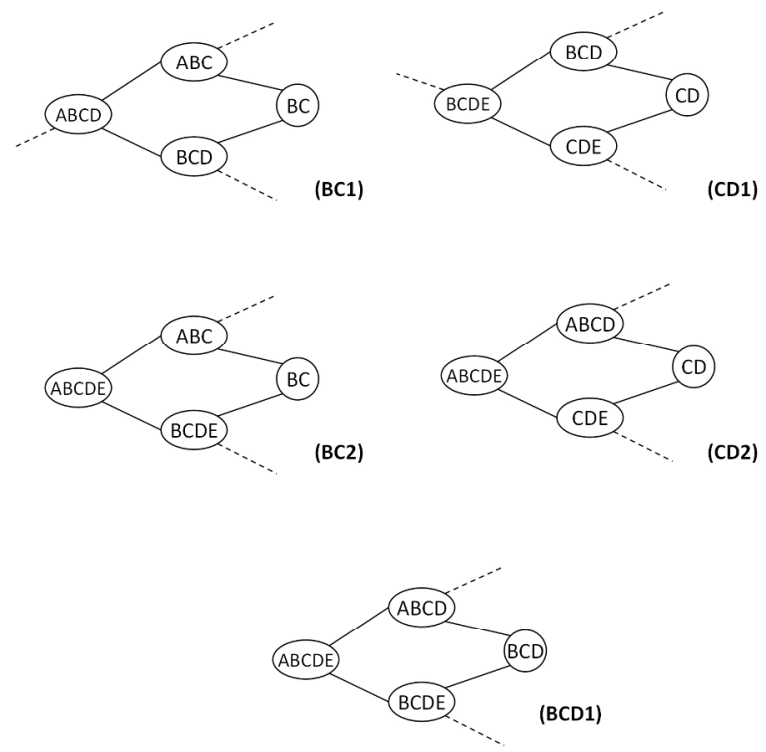

Sequences of states that can produce a DWC in a 5 component mixture. $190 \times 254 \mathrm{~mm}(300 \times 300$ DPI $)$ 


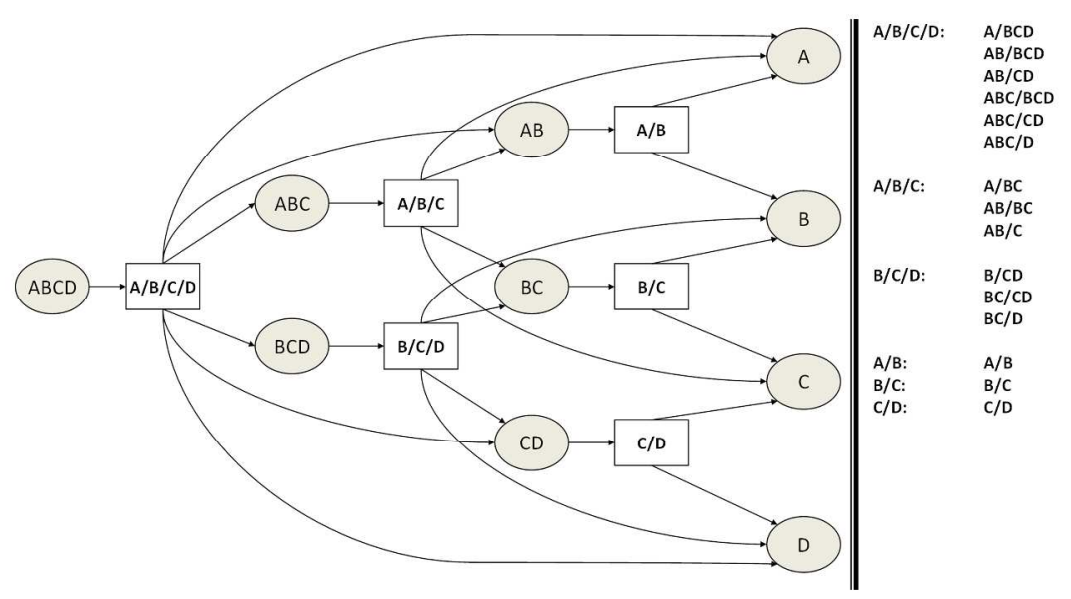

Two equivalent superstructures for a 4 component separation. $190 \times 254 \mathrm{~mm}(300 \times 300 \mathrm{DPI})$ 

$254 \times 190 \mathrm{~mm}(300 \times 300 \mathrm{DPI})$ 
Example of how the flows in some sections must be modified to satisfied the mass balances. $254 \times 190 \mathrm{~mm}(300 \times 300 \mathrm{DPI})$ 


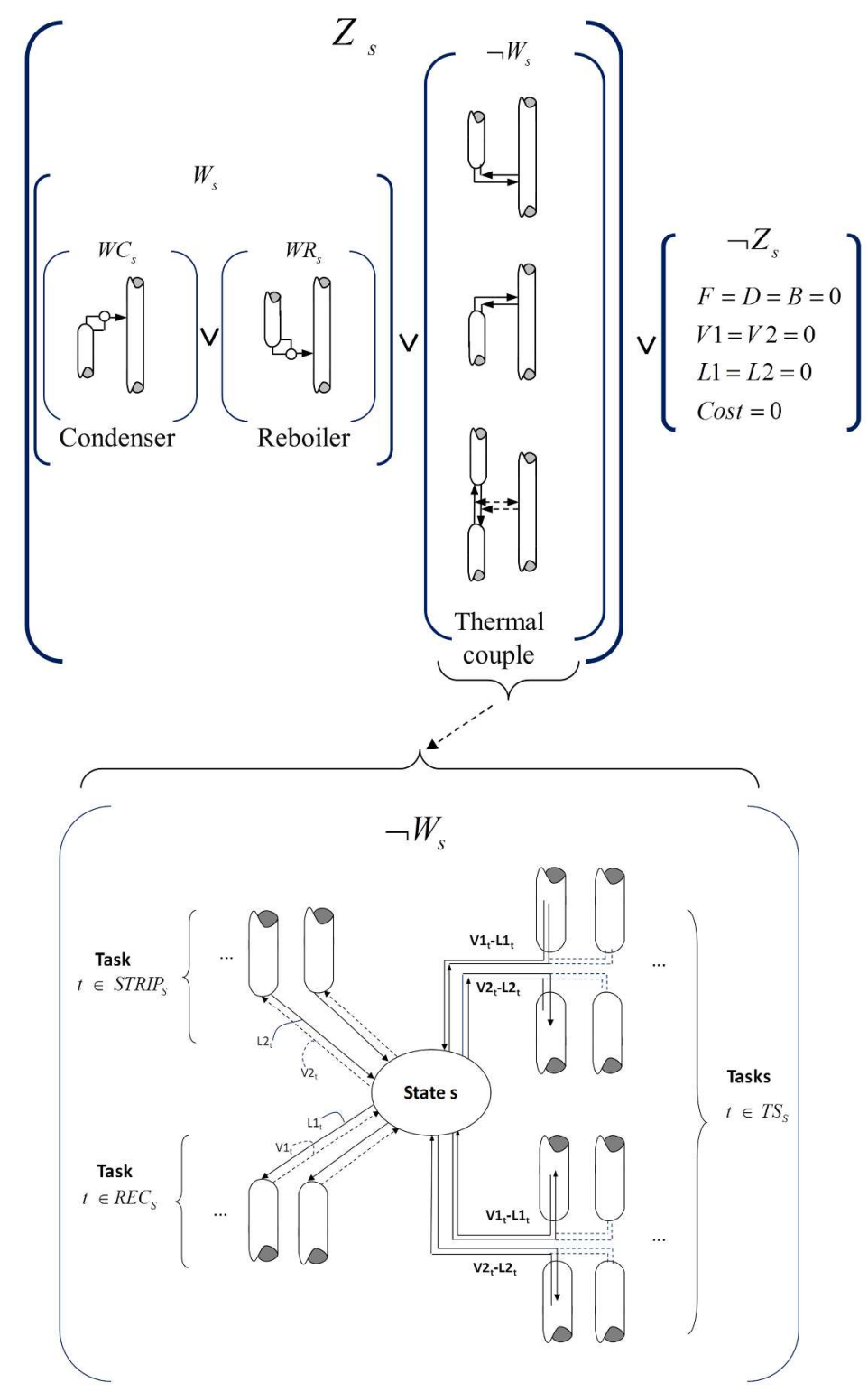

Conceptual graphical description of disjunction D-2 (see text). $190 \times 254 \mathrm{~mm}(300 \times 300$ DPI) 


1
2
3
4
5
6
7
8
9
10
11
12
13
14
15
16
17
18
19
20
21
22
23
24
25
26
27
28
29
30
31
32
33
34
35
36
37
38
39
40
41
42
43
44
45
46
47
48
49
50
51
52
53
54
55
56
57
50

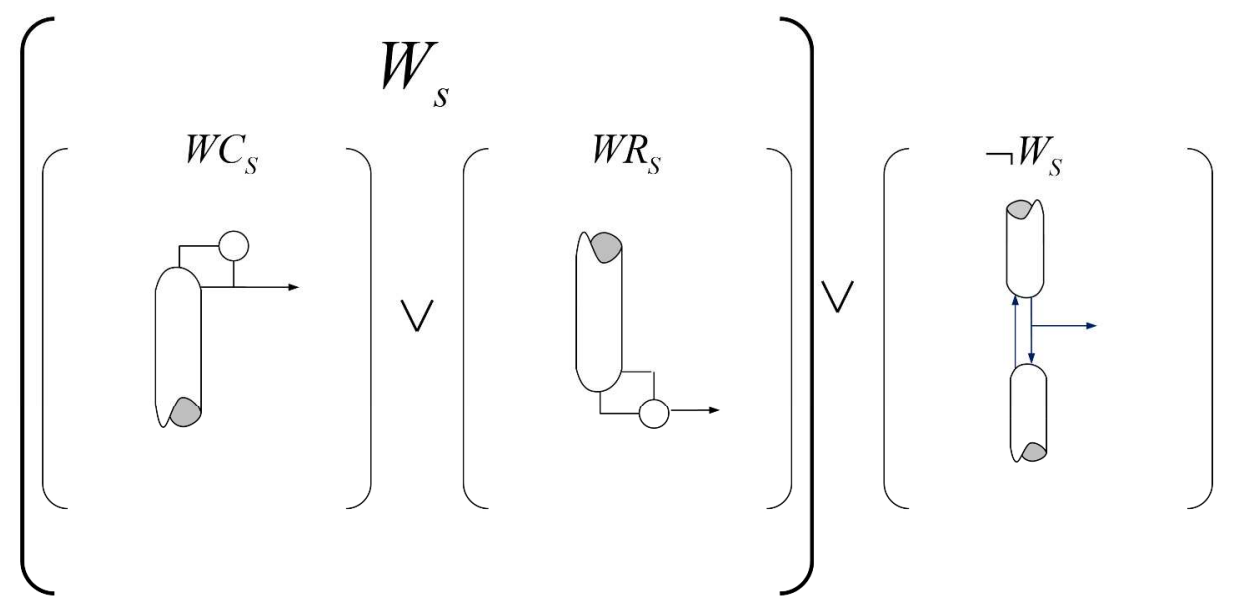

Conceptual graphical representation of disjunction D-3 (see text). $254 \times 190 \mathrm{~mm}$ (300 x 300 DPI) 
Bare cost module of the vertical vessel as a function of its volume $254 \times 190 \mathrm{~mm}$ (300 x 300 DPI) 
Best solution for example1 (sol 1.1). Sequence of tasks and states and one out of the four thermodynamically equivalent possible arrangements in actual columns $190 \times 254 \mathrm{~mm}(300 \times 300 \mathrm{DPI})$ 

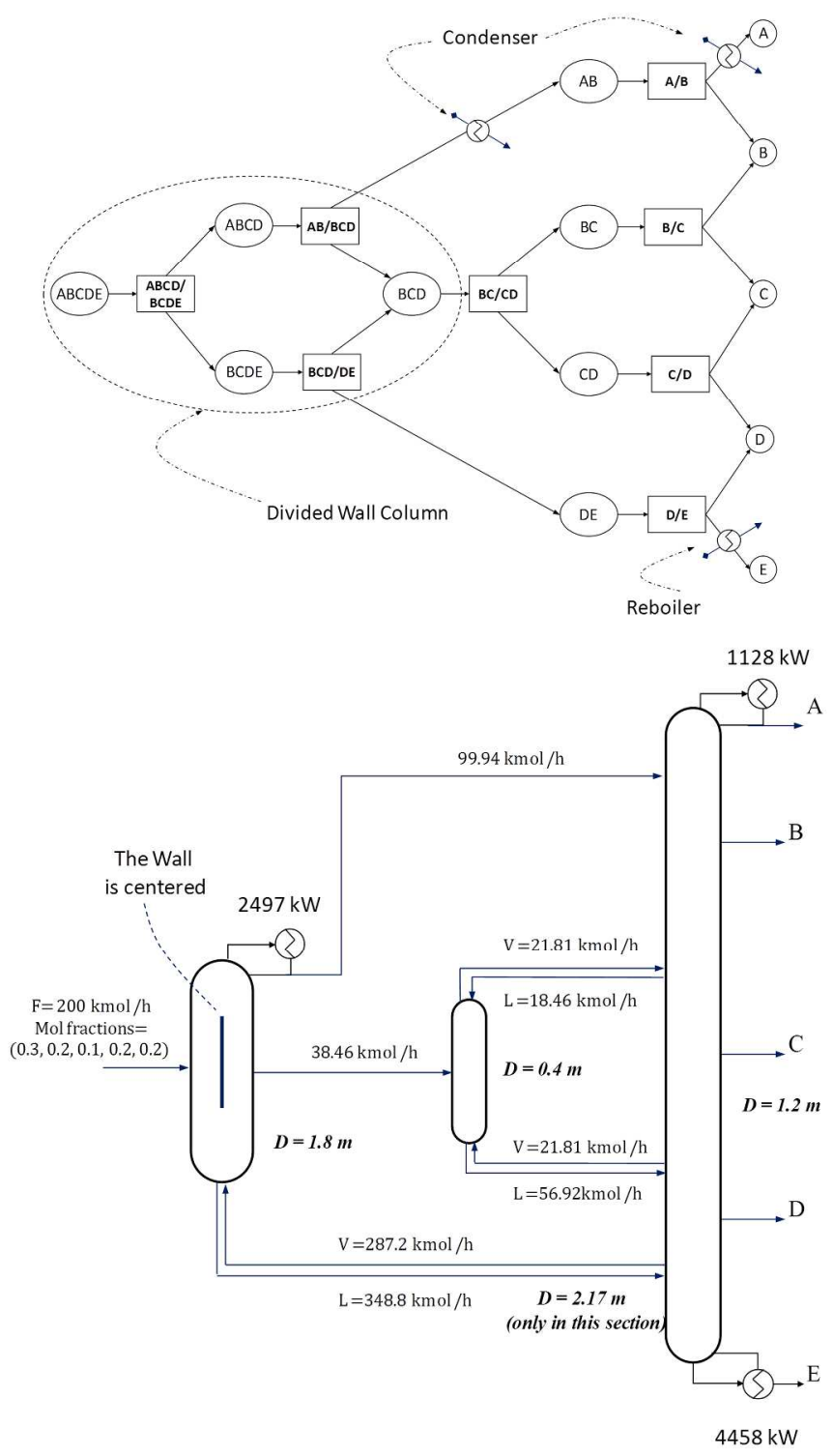

Second best solution for example 1. (sol 1.3). $190 \times 254 \mathrm{~mm}(300 \times 300$ DPI $)$ 
Best solution for example 1 including a DWC that is not thermally coupled with the rest of the system (sol $1.4)$. $190 \times 254 \mathrm{~mm}(300 \times 300 \mathrm{DPI})$ 

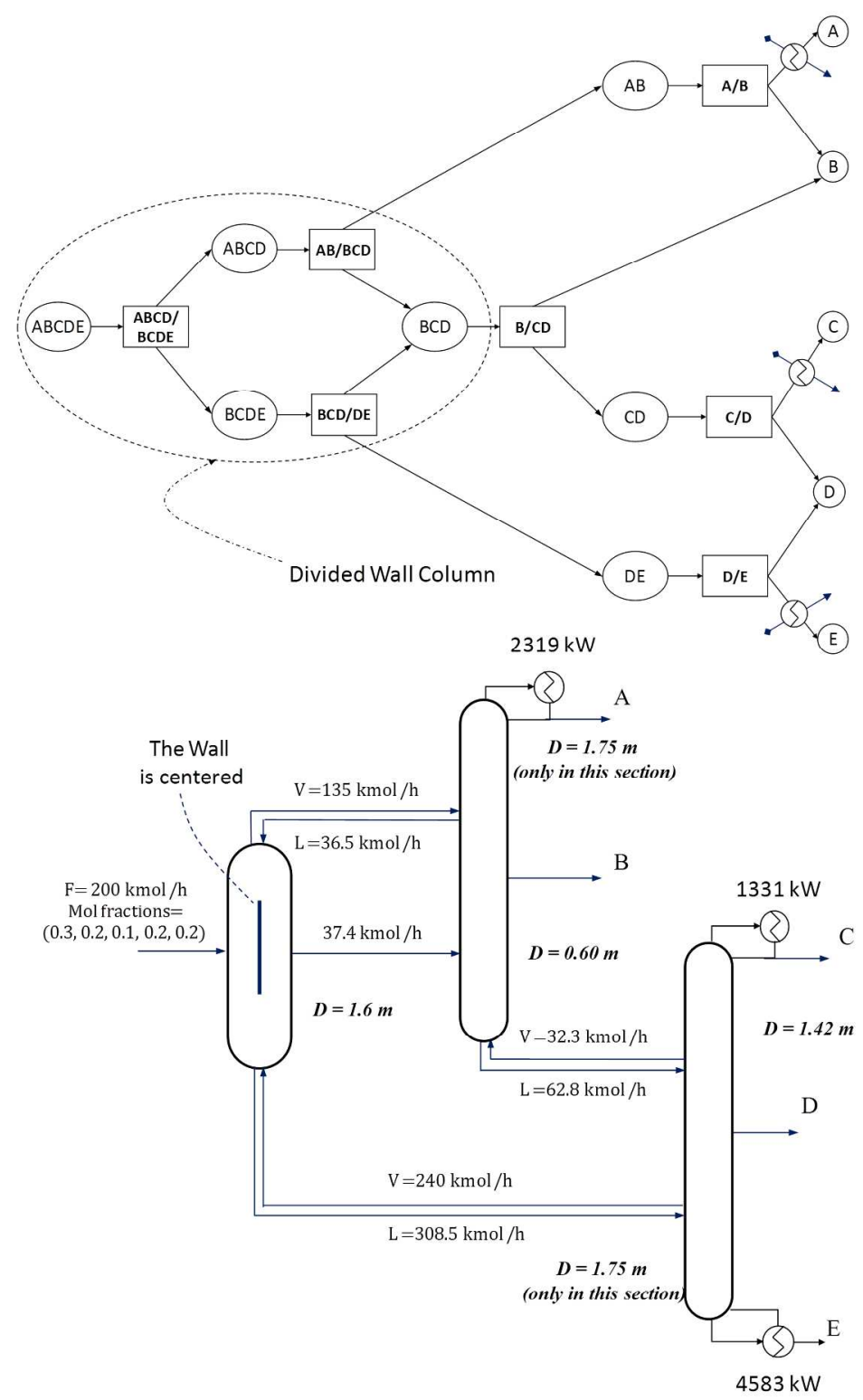

Best solution found using the heuristic algorithm in example 1 (sol 1.5). $190 \times 254 \mathrm{~mm}(300 \times 300 \mathrm{DPI})$ 
Best solution for example 1, using only conventional columns, "classical column sequencing". (Sol 1.6) $254 \times 190 \mathrm{~mm}(300 \times 300 \mathrm{DPI})$ 


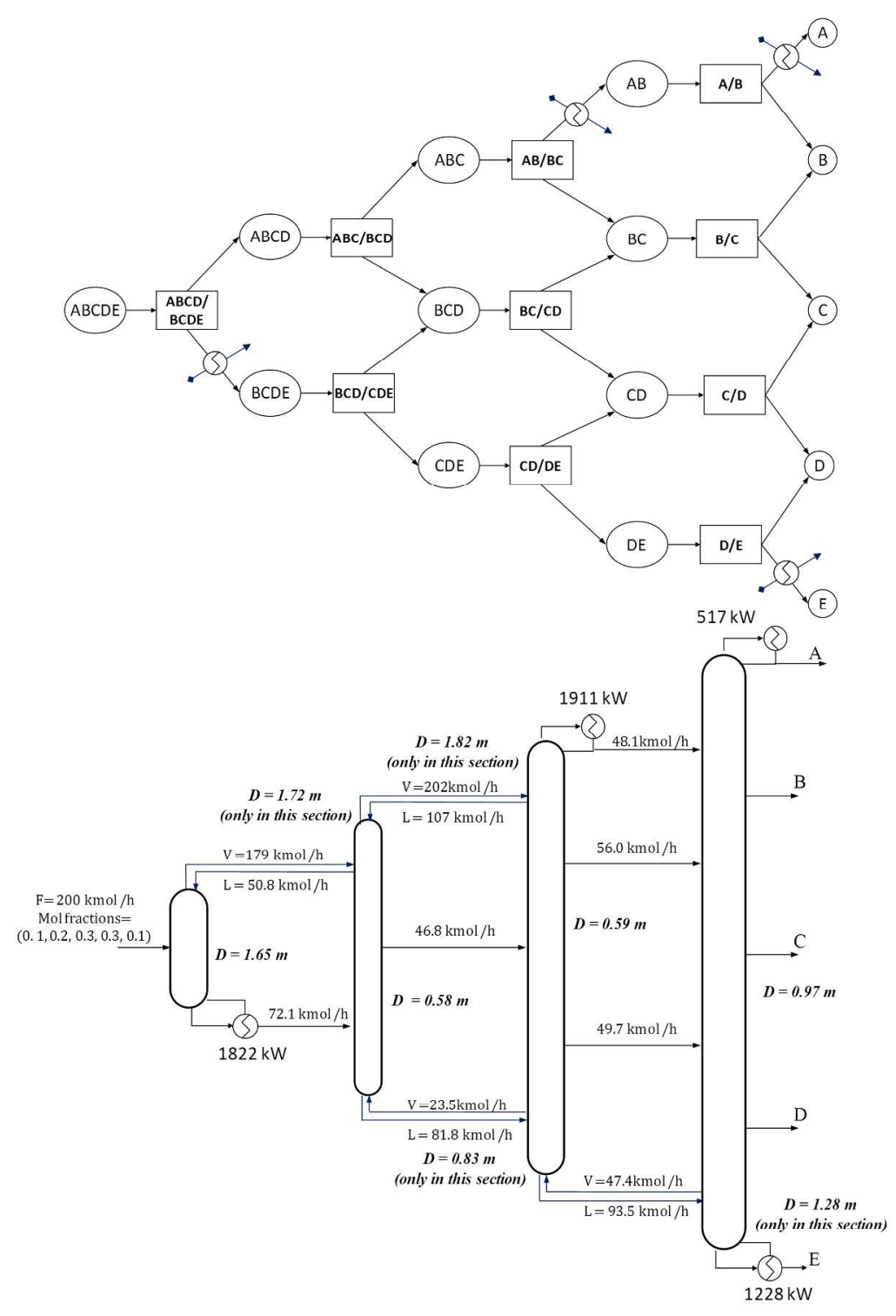

Optimal solution in example 2. (Sol 2.1) $190 \times 254 \mathrm{~mm}(300 \times 300$ DPI $)$ 


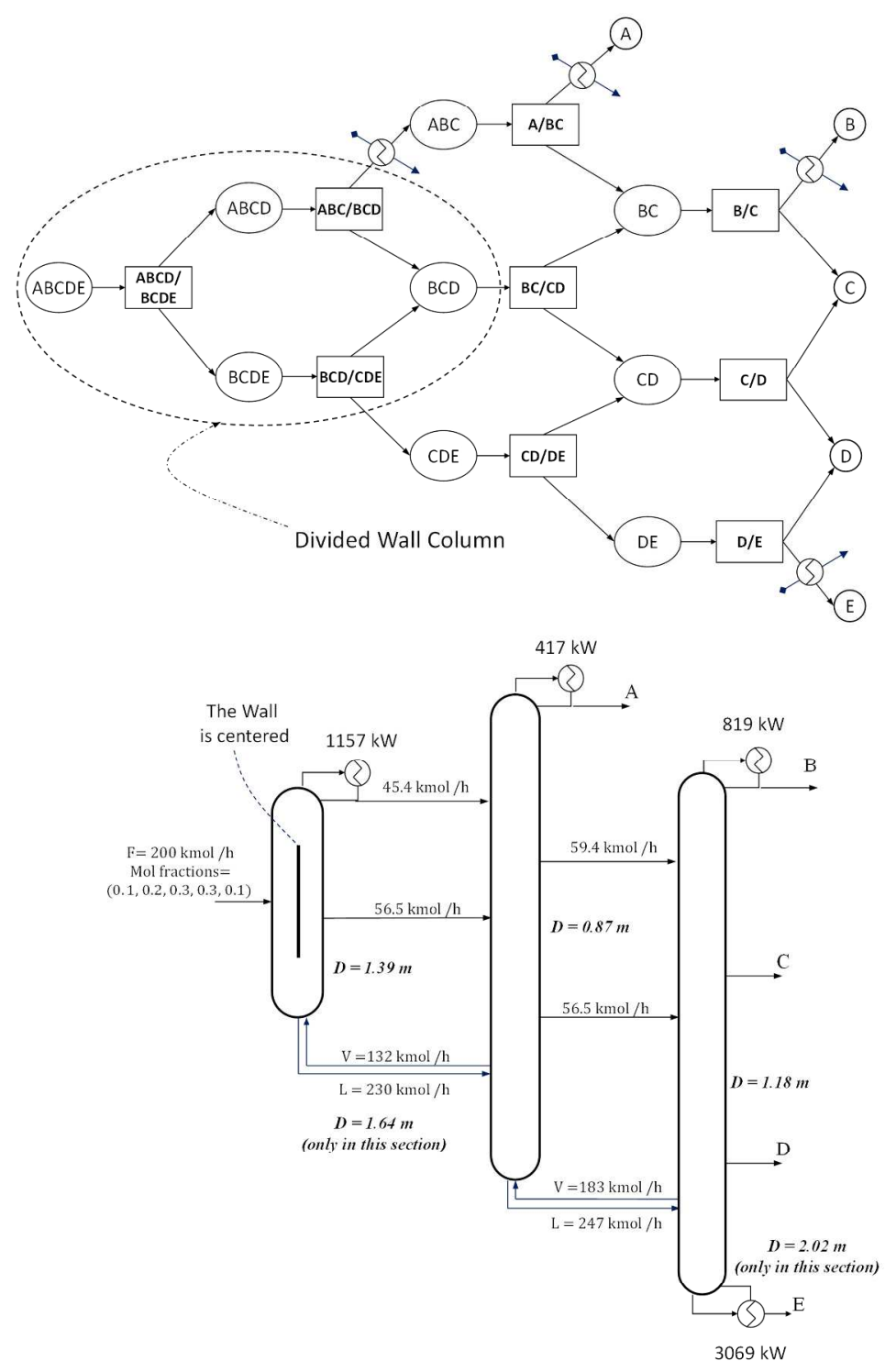

Best solution for example 2 using the heuristic algorithm. (Sol 2.2) $190 \times 254 \mathrm{~mm}(300 \times 300 \mathrm{DPI})$ 
Best solution for example 2, using only conventional columns, "classical column sequencing". (Sol 2.3). $254 \times 190 \mathrm{~mm}(300 \times 300$ DPI $)$ 
1

2

3

4

5

6

7

8

9

10

11

12

13

14

15

16

17

18

19

20

21

22

23

24

25

26

27

28

29

30

31

32

33

34

35

36

37

38

39

40

41

42

43

44

45

46

47

48

49

50

51

52

53

54

55

56

57

58

59

60

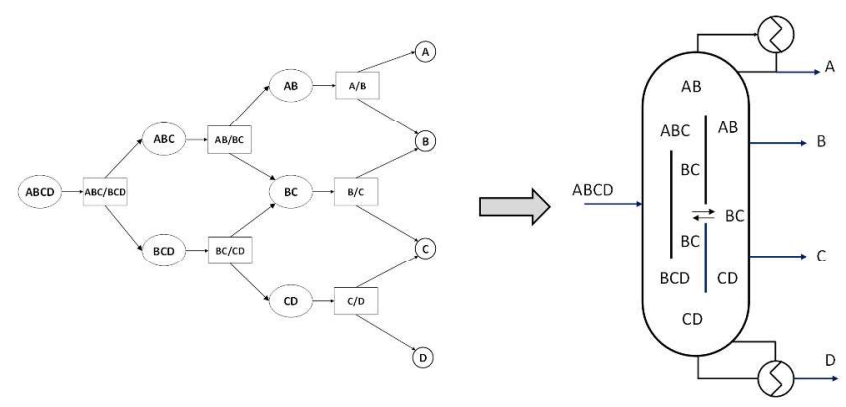

(a)

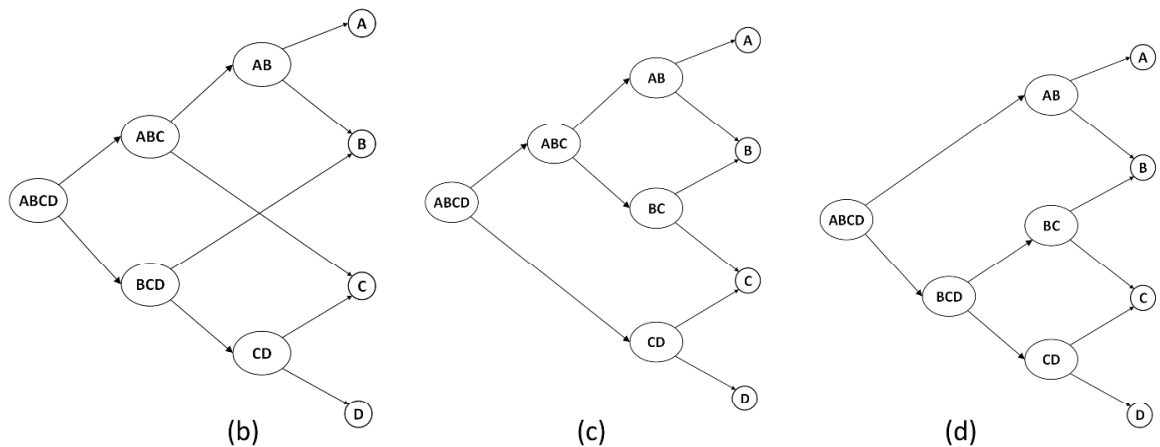

(b)

(c)

(d)

Fully thermally coupled sequences of task for the separation of a 4 component mixture. (a) maximum number of separation tasks $(\mathrm{N}(\mathrm{N}-1) / 2=6)$ and an arrangement using two internal walls. (b, c, d) Sequences using the minimum number of separation tasks $((4 \mathrm{~N}-6) / 2=5)$. 
The two feasible arrangements using two internal walls for 4 component mixtures with the minimum number of separation tasks $254 \times 190 \mathrm{~mm}(300 \times 300$ DPI $)$ 
Recursive approach for generating an arrangement of multiple internal walls for a 5 component separation sequence $(a-c)$. (d) sequence of states and tasks, (e) and (f) two possible arrangements of the internal wall in an actual column. $254 \times 190 \mathrm{~mm}(300 \times 300 \mathrm{DPI})$ 